\title{
NOVIKOV FUNDAMENTAL GROUP
}

\author{
JEAN-FRANÇOIS BARRAUD ${ }^{+}$, AGNÈS GADBLED ${ }^{++}$, ROMAN GOLOVKO ${ }^{+++}$, \\ AND HÔNG VÂN LÊ $\hat{E}^{++++}$
}

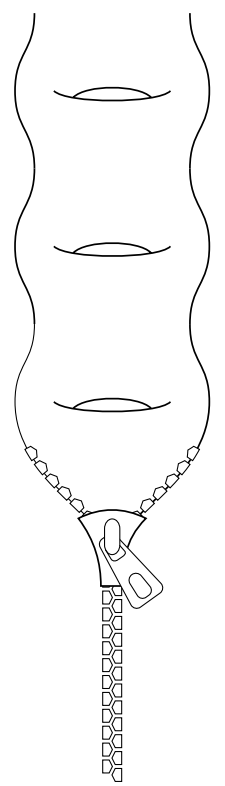

\begin{abstract}
Given a 1-cohomology class $u$ on a closed manifold $M$, we define a Novikov fundamental group associated to $u$, generalizing the usual fundamental group in the same spirit as Novikov homology generalizes Morse homology to the case of non exact 1-forms.

As an application, lower bounds for the minimal number of index 1 and 2 critical points of Morse closed 1-forms are obtained, that are different in nature from those derived from the Novikov homology.
\end{abstract}

Date: June 26, 2018.

2010 Mathematics Subject Classification. Primary 57R19; Secondary 57R70, 57R17.

+ This work was partially supported by the CIMI.

++ This work was partially supported by the Hausdorff Research Institute for Mathematics (HIM), University of Bonn and Wallenberg grant KAW 2016-0440.

+++ This work was supported by the ERC Consolidator Grant 646649 "SymplecticEinstein".

++++ Research of HVL was supported by RVO:67985840 and the GAČR-project 1800496S. 


\section{INTRODUCTION AND MAIN RESULTS}

Consider a smooth and closed manifold $M$, and a closed 1-form $\alpha$ in a non trivial cohomology class $u \in H^{1}(M, \mathbb{R})$.

By analogy with the exact case, a point $p$ in $M$ is called critical for $\alpha$ if $\alpha_{p}=0$. Such a critical point $p$ is said to be non degenerate, or Morse, if it is non degenerate as the critical point of a local primitive $f_{\alpha}$ of $\alpha$ in a neighborhood of $p$. The 1-form $\alpha$ itself is said to be Morse if all its critical points are non degenerate.

The Novikov theory relates the critical points of (Morse) 1 -forms $\alpha$ in the cohomology class $u$ and the "topology" of the pair $(M, u)$.

The case $u=0$ is addressed by the Morse theory, which exhibits a tight relation between the topology of $M$ and the critical points of a Morse function on it ; in particular, Morse functions have to have enough critical points to generate both $H_{*}(M)$ (the classical Morse inequality) and $\pi_{1}(M)$ ([Sharko1993, Theorem 7.10, p. 172]).

Another extreme case is when $M$ fibers over the circle $M \stackrel{\pi}{\rightarrow} \mathbb{S}^{1}$ and $\alpha=\pi^{*}(d \theta): \alpha$ obviously has no critical point in this case, regardless of the actual topology of $M$. Notice that the converse is also true : if there is a 1-form without critical points in $u$, then it comes from a fibration over the circle [Tischler1970].

The celebrated Novikov homology Novikov1981] offers some algebraic measurement of the complexity of the topology of $M$ "with respect to $u$ " or of the pair $(M, u)$, and the Novikov inequalities [Novikov1981] as well as further numerical invariants developed in the same spirit Farber2004, Pajitnov2006 give lower bounds for the number of critical points of Morse 1 -forms in the cohomology class $u$. As consequence, Novikov proved the existence of periodic solution in Kirchhoff problem DNF1984, Theorem 1, Appendix 1, p. 386]. Novikov homology also enters in theory of symplectic fixed points on compact symplectic manifolds [LO1995] or Lagrangian intersections/embeddings problems ([Sikorav1986, Damian2009, Gadbled2009]).

The object of this paper is to give a Novikov theoretic version of the fundamental group, that plays in Novikov theory the role the usual fundamental group plays in Morse theory.

More precisely, we fix a non trivial cohomology class $u \in H^{1}(M, \mathbb{R})$, and consider an integration cover $\tilde{M}$ associated to $u$.

Remark 1. Throughout this paper, all the integration covers will always be supposed to be connected.

The usual choices of covering spaces are either the minimal integration cover, or the universal cover, but any intermediate cover could be used : each choice defines a different version of the invariant. 
The situation is similar to Novikov homology, which was originally defined using the minimal integration cover [Novikov1981 but was later extended to the universal cover (and in fact any intermediate cover) by Sikorav Sikorav1987.

We denote by $\tilde{u}: \pi_{1}(M) \rightarrow \mathbb{R}$ the composition of the Hurewicz homomorphism $\pi_{1}(M) \rightarrow H_{1}(M, \mathbb{Z})$ with the evaluation map $u: H_{1}(M, \mathbb{Z}) \rightarrow \mathbb{R}$. Since $\pi_{1}(\tilde{M}) \subset \operatorname{ker} \tilde{u}$, the homomorphism $\tilde{u}$ descends to a homomorphism also denoted by $u: \mathcal{D}=\pi_{1}(M) / \pi_{1}(\tilde{M}) \rightarrow \mathbb{R}$. Note that $\mathcal{D}$ is the deck transformation group of $\tilde{M}$.

Then to $u$ and each choice of integration cover $\tilde{M}$ is associated a group $\pi_{1}(\tilde{M}, u)$ endowed with an action of $\mathcal{D}$, such that the following holds :

Theorem 1.1. (1) For every choice of a closed 1-form $\alpha$ in the class $u$ and a primitive $f_{\alpha}$ of $\alpha$, there is a group $\pi_{1}\left(f_{\alpha}\right)$ that is isomorphic to $\pi_{1}(\tilde{M}, u)$,

(2) there is a suitable notion of generators and relations "up to deck transformations and a completion" for which $\pi_{1}(\tilde{M}, u)$ is finitely presented,

(3) if $\alpha$ is Morse, then the minimal number of generators (up to deck transformations and a completion) of $\pi_{1}(\tilde{M}, u)$ is a lower bound for the number of index 1 critical points of $\alpha$,

(4) similarly, if $\alpha$ is Morse, then the minimal number of relations (up to deck transformations and a completion) in $\pi_{1}(\tilde{M}, u)$ is a lower bound for the number of index 2 critical points of $\alpha$.

Remark 2. This definition does not use any extra choice of a base point. This is because in the non exact case (i.e. $u \neq 0$ ), which is the case we are interested in, there is a canonical choice of a base point, which essentially consists in putting it "at $-\infty$ ".

In the exact case however, the primitives of $u$ are all bounded and this choice does not make sense anymore.

Using other conventions about the base point to get a more uniform definition is possible but restrictive : the usual notion of base point could be used, but breaks the action of the deck transformations which is an essential feature of the resulting group, or it could be replaced by a more subtle notion of base point (like properly embedded and shift invariant lines $\mathbb{R} \rightarrow \tilde{M}$ ) but this requires $u$ to be integral.

Since we are only interested in the non exact case, we will stick to it and use the canonical choice of base point coming with it throughout this paper.

To express the functoriality properties of the construction, consider the Novikov fundamental group as associated to objects of the form $(\tilde{M} \stackrel{\pi}{\rightarrow}$ $M, u)$, where $\tilde{M}$ is a connected covering space of a closed smooth manifold $M$ and $u$ is a non trivial cohomology class in $H^{1}(M, \mathbb{R})$ satisfying $\pi^{*}(u)=0$. 
A morphism between two such objects $\left(\tilde{M}_{i} \stackrel{\pi_{i}}{\longrightarrow} M_{i}, u_{i}\right), i=1,2$, is then a smooth covering map

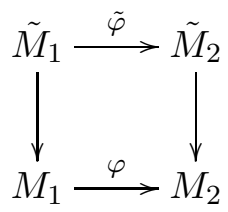

such that

$$
u_{1}=\varphi^{*}\left(u_{2}\right) .
$$

Theorem 1.2. The Novikov fundamental group is functorial, i.e.

(1) to an arrow $\left(\tilde{M}_{1} \rightarrow M_{1}, u_{1}\right) \stackrel{\varphi}{\rightarrow}\left(\tilde{M}_{2} \rightarrow M_{2}, u_{2}\right)$ as above is associated a group homomorphism $\varphi_{*}: \pi_{1}\left(\tilde{M}_{1}, u_{1}\right) \rightarrow \pi_{1}\left(\tilde{M}_{2}, u_{2}\right)$ which commutes with the corresponding deck transformations,

(2) if $\left(\tilde{M}_{2} \rightarrow M_{2}, u_{2}\right) \stackrel{\phi}{\rightarrow}\left(\tilde{M}_{3} \rightarrow M_{3}, u_{3}\right)$ is another morphism, then $[\phi \circ \varphi]_{*}=\phi_{*} \circ \varphi_{*}$.

Notice that if $\left(\tilde{M}_{2} \rightarrow M_{2}, u_{2}\right)$ is given, and $M_{1} \stackrel{\varphi}{\rightarrow} M_{2}$ is a map from a smooth closed manifold $M_{1}$ to $M_{2}$, then $\varphi^{*}\left(\tilde{M}_{2}\right)$ is an integration cover of $\varphi^{*}\left(u_{2}\right)$, but it does not need to be connected (nor needs $\varphi^{*}\left(u_{2}\right)$ to be non trivial). However, we have the following proposition :

Proposition 1.3. In the situation above, each connected component $\varphi^{*}\left(\tilde{M}_{2}\right)_{0}$ of $\varphi^{*}\left(\tilde{M}_{2}\right)$ is an integration cover for $\varphi^{*}\left(u_{2}\right)$. Whenever $\varphi^{*}\left(u_{2}\right) \neq 0$, the group $\pi_{1}\left(\varphi^{*}\left(\tilde{M}_{2}\right)_{0}, \varphi^{*} u_{2}\right)$ does not depend on the choice of connected component and $\varphi$ induces a group morphism

$$
\pi_{1}\left(\varphi^{*}\left(\tilde{M}_{2}\right)_{0}, \varphi^{*} u_{2}\right) \stackrel{\varphi_{*}}{\longrightarrow} \pi_{1}\left(\tilde{M}_{2}, u_{2}\right) .
$$

Another feature of this Novikov fundamental group is that it supports a Hurewicz morphism :

Theorem 1.4. Suppose that $\tilde{M}$ is the minimal integration cover.

Then there is a surjective homomorphism

$$
\mathfrak{h}: \pi_{1}(\tilde{M}, u) \rightarrow H N_{1}(M, u ; \mathbb{Z}) .
$$

whose kernel is a suitable abelianization $\pi_{1}(\tilde{M}, u)^{\frac{\mathrm{ab}}{}}$ that takes the completion process into account (see section 4).

Moreover, the following example shows that this invariant is non trivial, and different in nature from the invariants derived from the Novikov homology :

Theorem 1.5. Let $S$ be the Poincaré homology sphere and $M=\mathbb{T}^{n} \sharp(S \times$ $\left.\mathbb{S}^{n-3}\right)$ be the connected sum of a torus and the product of $S$ with a sphere.

On $M$, consider the class $u=\pi^{*}\left(d \theta_{1}\right)$ where $\pi$ is the projection $M \stackrel{\pi_{1}}{\longrightarrow} \mathbb{S}^{1}$ to the first coordinate $\theta_{1}$ on the torus. 
Then the Novikov homology associated to the minimal integration cover of $u$ vanishes in degrees 1 and 2, but the associated Novikov fundamental group $\pi_{1}(\tilde{M}, u)$ is non trivial. Its minimal number of generators and relations up to deck transformations and completion are 2 and 2.

In particular, any Morse 1-form $\alpha$ in the class $u$ necessarily has at least 2 index 1 and 2 index 2 critical points.

Remark 3. The Poincaré sphere does not play a crucial role here except for the explicit bounds 2 and $2: S \times \mathbb{S}^{n-3}$ could be replaced by any closed $n$ manifold $X, n \geq 4$, such that $H_{1}(M, \mathbb{Z})=H_{2}(M, \mathbb{Z})=0$, and the Novikov fundamental group would remain non trivial, while the Novikov homology would still vanish.

Notice that the Novikov homology $H N_{*}\left(M, u ; \pi_{1}(M)\right)$ associated to the universal cover of $M$ (i.e. when $\mathcal{D}=\pi_{1}(M)$ ) contains much more information about the fundamental group than that associated to the minimal integration cover. In particular, $H N_{1}\left(M, u ; \pi_{1}(M)\right) \neq 0$ in this example. However, the second Novikov homology group $H N_{2}\left(M, u ; \pi_{1}(M)\right)$ still vanishes, so it does not give any constrain on the number of index 2 critical points (while the Novikov fundamental group does).

Remark 4. A nice observation (see beginning of section 5.3.2) pointed to us by A. Pajitnov allows to produce index 2 critical points out the comparison of the Novikov homologies associated to the universal and the minimal covers, even though $H N_{2}(M, u)=H N_{2}\left(M, u ; \pi_{1}(M)\right)=0$.

However, a similar example $\left(\mathbb{T}^{n} \sharp \mathbb{R} \mathbb{P}^{n}\right.$, see section 5.3.2) shows that the estimates derived from the Novikov fundamental group are also essentially different from those obtained in this way.

The construction of the Novikov fundamental group in this paper is a natural adaptation to the homotopy setting of the interpretation of the Novikov complex (and the Novikov Homology itself as well in many cases, see [Sikorav1987] and [Usher2008]) as the projective limit of chain complexes relative to sublevels.

A very similar definition appears for higher homotopy groups in [FGS2010], but the fundamental group is not discussed, nor a fortiori the number of generators and relations and their relation to critical points of Morse 1-forms in the class $\alpha$.

In Latour1994, Latour defines several spaces that are interesting to compare to the present construction. First, he considers (Latour1994, 5.7, p. 184]) the group $\pi_{1}^{\text {Latour }}(u)$ of loops that can be "slid to $-\infty$ " in the minimal integration cover. Despite similar notations, this group is somewhat orthogonal to our construction since such loops are automatically trivial in our construction. The closest notion considered by Latour is the $\pi_{0}$ of the space of "paths going to $-\infty$ ". The Novikov fundamental group we define here can be thought of as a Novikov completion of the group generated by this space. 
The paper is organized as follows : the first section is this introduction, the second section is devoted to the construction of the Novikov fundamental group and the definition of a suitable notion of generators and relations, the third to the Morse interpretation of it and the proof of Theorem 1.1, the fourth to the discussion of a Novikov version of the Hurewicz morphism, and finally the last one to the discussion of an example and the proof theorem 1.5 .

\section{NOVIKOV FUndAMENTAL GROUP}

2.1. Projective limit with respect to sublevels. Let $M$ be a closed smooth manifold, $u \in H^{1}(M, \mathbb{R})$ a non trivial cohomology class, and $\alpha \in u$ a closed 1-form in the class $u$.

Let $p: \tilde{M} \rightarrow M$ be an integration cover of $u$ (see Remark 11) and $\mathcal{D}=$ $\pi_{1}(M) / \pi_{1}(\tilde{M})$ the associated deck transformation group.

Notice that for the minimal integration cover, we have $\pi_{1}(\tilde{M})=\operatorname{ker} u$, and $\mathcal{D}=\mathbb{Z}^{k}$, where $k$ is the irrationality degree of $u$. In particular, $k=1$ if $u$ is integral, i.e. if $u \in H^{1}(M, \mathbb{Z})$.

The 1 -form $p^{*}(\alpha)$ on $\tilde{M}$ is exact, and we pick a primitive $f_{\alpha}: \tilde{M} \rightarrow \mathbb{R}$. For $h \in \mathbb{R}$, we let

$$
\begin{gathered}
\tilde{M}^{\leq h}=\left\{p \in \tilde{M}, f_{\alpha}(p) \leq h\right\} \\
\lfloor\tilde{M}\rfloor_{h}=\tilde{M} / \tilde{M}^{\leq h}=\tilde{M} / \sim
\end{gathered}
$$

where $\sim$ collapses $\tilde{M}^{\leq h}$ to a point $: p \sim q \Leftrightarrow f_{\alpha}(p) \leq h$ and $f_{\alpha}(q) \leq h$. This space comes with a natural base point $\star_{h}$, given by the collapsed sublevel $\tilde{M}^{\leq h}$.

We now consider the family of groups :

$$
\left\lfloor\pi_{1}\left(f_{\alpha}\right)\right\rfloor_{h}=\pi_{1}\left(\lfloor\tilde{M}\rfloor_{h}, \star_{h}\right) .
$$

Inclusions of sublevels induce natural maps for any $h, h^{\prime} \in \mathbb{R}$ with $h<h^{\prime}$ :

$$
\left\lfloor\pi_{1}\left(f_{\alpha}\right)\right\rfloor_{h} \stackrel{\zeta_{h}^{h^{\prime}}}{\longrightarrow}\left\lfloor\pi_{1}\left(f_{\alpha}\right)\right\rfloor_{h^{\prime}}
$$

Moreover, these maps are compatible with successive inclusions : if $h, h^{\prime}, h^{\prime \prime} \in$ $\mathbb{R}$ are such that $h<h^{\prime}<h^{\prime \prime}$, then the following diagram is commutative :

$$
\left\lfloor\pi_{1}\left(f_{\alpha}\right)\right\rfloor_{h} \stackrel{\zeta_{h}^{h^{\prime}}}{\longrightarrow}\left\lfloor\pi_{1}\left(f_{\alpha}\right)\right\rfloor_{h^{\prime}} \stackrel{\zeta_{h^{\prime}}^{h^{\prime \prime}}}{\longrightarrow}\left\lfloor\pi_{1}\left(f_{\alpha}\right)\right\rfloor_{h^{\prime \prime}} .
$$

As a consequence the projective limit when $h$ goes to $-\infty$ is well defined :

Definition 2.1. Define the Novikov fundamental group associated to $\alpha$ as the projective limit

$$
\pi_{1}\left(f_{\alpha}\right)=\varliminf_{h} \lim _{h}\left\lfloor\pi_{1}\left(f_{\alpha}\right)\right\rfloor_{h} .
$$


As a projective limit, this group comes with maps $\zeta_{\infty}^{h}$ to the "zipped" groups :

$$
\pi_{1}\left(f_{\alpha}\right) \stackrel{\zeta_{\infty}^{h}}{\longrightarrow}\left\lfloor\pi_{1}\left(f_{\alpha}\right)\right\rfloor_{h} .
$$

and each element $g$ has a minimal height, $h_{f_{\alpha}}(g)$, with respect to $f_{\alpha}$, defined as

$$
h_{f_{\alpha}}(g)=\inf \left\{h \in \mathbb{R}, g \in \operatorname{ker} \zeta_{\infty}^{h}\right\} .
$$

Remark 5. The fact that an element $g$ appears to be trivial above a level $a_{0}$ (i.e. $\zeta_{\infty}^{a}(g)=1$ ) does not mean in general that for levels $a<a_{0}, g$ can be represented in $\left\lfloor\pi_{1}\left(f_{\alpha}\right)\right\rfloor_{a}$ by a loop that stays below level $a_{0}$.

For instance, if $\gamma$ and $\gamma^{\prime}$ are two paths running from and to $-\infty$, such that for some levels $a_{-}<a_{0}<a_{+}$, we have

- $\gamma \neq 1$ in $\left\lfloor\pi_{1}\left(f_{\alpha}\right)\right\rfloor_{a_{+}}$

- $\gamma^{\prime}=1$ in $\left\lfloor\pi_{1}\left(f_{\alpha}\right)\right\rfloor_{a_{0}}$ but $\gamma^{\prime} \neq 1$ in $\left\lfloor\pi_{1}\left(f_{\alpha}\right)\right\rfloor_{a_{-}}$,

then the element $g$ defined by $\gamma \gamma^{\prime} \gamma^{-1}$ is trivial in $\left\lfloor\pi_{1}\left(f_{\alpha}\right)\right\rfloor_{a_{0}}$, but representing its homotopy class in $\lfloor\tilde{M}\rfloor_{a_{-}}$may require a path going above level $a_{+}$(see the middle picture in Figure 10.
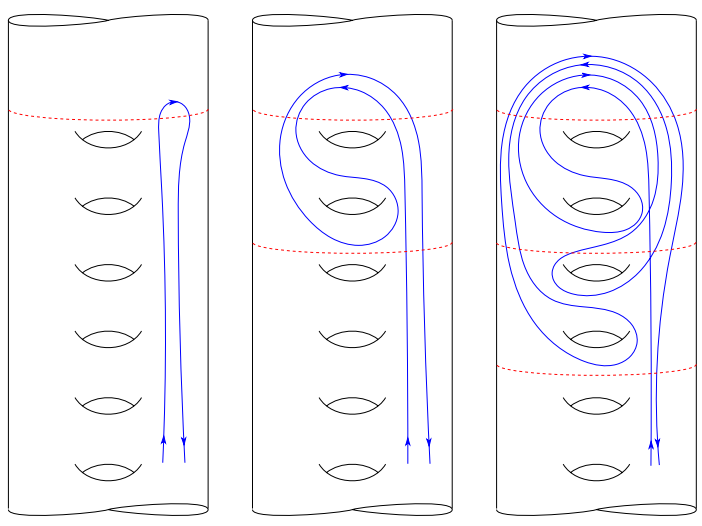

FiguRE 1. An element in $\pi_{1}(\tilde{M}, u)$ whose deeper and deeper representatives do not eventually become constant above a given level

In particular, if a path $\mathbb{R} \stackrel{\gamma}{\rightarrow} \tilde{M}$ such that $\lim _{t \rightarrow \pm \infty} f_{\alpha}(\gamma(t))=-\infty$ clearly defines an element in $\pi_{1}(\tilde{M}, u)$, not all the elements can be described in this way.

Moreover, among such paths, some are better than the others, in that they do not go down and up too badly :

Definition 2.2. An element $g$ in $\pi_{1}\left(f_{\alpha}\right)$ will be said to be clean if it can be defined by a path $\mathbb{R} \stackrel{\gamma}{\rightarrow} \tilde{M}$ with $\lim _{t \rightarrow \pm \infty} f_{\alpha}(\gamma(t))=-\infty$ whose "wells have bounded depth", i.e. $\exists K>0, \forall\left(t_{1}, t_{2}\right) \in \mathbb{R}^{2}, \forall h \in \mathbb{R}$

$$
f_{\alpha}\left(\gamma\left(t_{1}\right)\right)=f_{\alpha}\left(\gamma\left(t_{2}\right)\right)=h \Rightarrow \forall t \in\left[t_{1}, t_{2}\right], f_{\alpha}(t) \geq h-K .
$$




\subsection{Invariance.}

Theorem 2.3. The group $\pi_{1}\left(f_{\alpha}\right)$ does not depend on the choice of the 1form $\alpha$ nor of the primitive $f_{\alpha}$.

More precisely, if $\alpha$ and $\beta$ are two forms in the same cohomology class $u \in \mathbb{H}^{1}(M, \mathbb{R}), f_{\alpha}$ and $f_{\beta}$ two primitives of $\alpha$ and $\beta$ on $\tilde{M}$, then there is a canonical isomorphism

$$
\pi_{1}\left(f_{\alpha}\right) \stackrel{\sim}{\rightarrow} \pi_{1}\left(f_{\beta}\right) .
$$

Definition 2.4. The identification of all these groups via these canonical isomorphism defines a group $\pi_{1}(\tilde{M}, u)$ which we call the Novikov fundamental group associated to $u$ (and the integration cover $\tilde{M}$ ).

Remark 6 . The proof of the invariance will in fact give a little more : the group itself is invariant, but it also comes with a preferred collection of projective systems defining it.

The argument below based on reciprocal inclusions of sublevels associated to different forms in the class $u$ will be reused several times and will be referred to as the invariance argument in the sequel, and we will say that projective systems satisfying the relation (2.1) for some constant $K$ are "essentially equivalent".

Proof. Since $\alpha-\beta$ is exact on $M$, there is a function $f$ on $M$ such that $f_{\beta}=f_{\alpha}+f \circ \pi$, where $\pi: \tilde{M} \rightarrow M$ is the projection. Since $M$ is compact, $f$ is bounded and there is a constant $K$ such that

$$
\forall p \in \tilde{M}, f_{\alpha}(p)-K \leq f_{\beta}(p) \leq f_{\alpha}(p)+K .
$$

As a consequence, for all $h \in \mathbb{R}$, we have the following inclusions of sublevels

$$
\tilde{M}^{f_{\alpha} \leq h-K} \subset \tilde{M}^{f_{\beta} \leq h} \subset \tilde{M}^{f_{\alpha} \leq h+K}
$$

which induce morphisms among relative fundamental groups, and make the following diagram commutative :

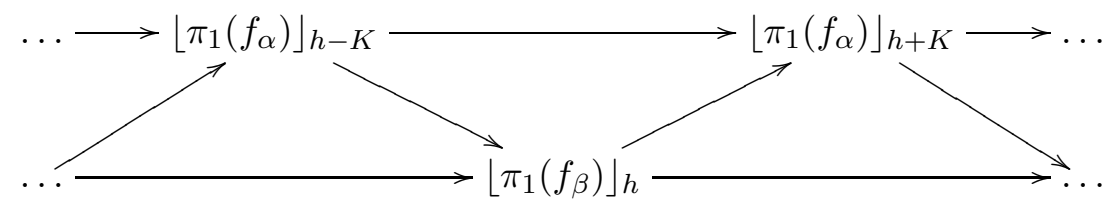

which in turn induce an isomorphism on the projective limit of the fundamental groups.

2.3. Deck transformations. For any $x \in \tilde{M}$ and any $\tau \in \mathcal{D}$ we have

$$
f_{\alpha}(\tau \circ x)=f_{\alpha}(x)+u(\tau)
$$

so that deck transformations send sublevels to sublevels :

$$
\tau \cdot \tilde{M}^{\leq h}=\tilde{M}^{\leq h+u(\tau)} \text {. }
$$


Hence $\tau$ also acts on $\pi_{1}\left(f_{\alpha}\right)$. As a consequence, for all $h \in \mathbb{R}$, we have an isomorphism

$$
\left\lfloor\pi_{1}\left(f_{\alpha}\right)\right\rfloor_{h} \stackrel{\tau}{\rightarrow}\left\lfloor\pi_{1}\left(f_{\alpha}\right)\right\rfloor_{h+u(\tau)} .
$$

This induces an isomorphism on the projective limit

$$
\pi_{1}\left(f_{\alpha}\right) \stackrel{\tau}{\rightarrow} \pi_{1}\left(f_{\alpha}\right)
$$

which finally defines an action of $\mathcal{D}$ on $\pi_{1}\left(f_{\alpha}\right)$.

2.4. Functoriality of the Novikov fundamental group. Here we prove theorem 1.2 ,

We start with an arrow $\left(\tilde{M}_{1} \rightarrow M_{1}, u_{1}\right) \stackrel{\varphi}{\rightarrow}\left(\tilde{M}_{2} \rightarrow M_{2}, u_{2}\right)$ i.e. with a commutative diagram

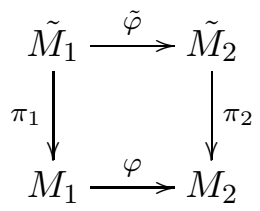

such that $\varphi^{*}\left(u_{2}\right)=u_{1}$.

Consider a 1 -form $\alpha_{2}$ in the class $u_{2}$ and a primitive $f_{2}$ of $\pi_{2}^{*} \alpha_{2}$ on $\tilde{M}_{2}$.

Then $f_{1}=\tilde{\varphi}^{*} f_{2}$ satisfies

$$
d f_{1}=d\left(\tilde{\varphi}^{*} f_{2}\right)=\tilde{\varphi}^{*} d f_{2}=\tilde{\varphi}^{*} \pi_{2}^{*} \alpha_{2}=\pi_{1}^{*} \varphi^{*} \alpha_{2},
$$

and hence is a primitive of $\pi_{1}^{*} \alpha_{1}$, where $\alpha_{1}=\varphi^{*} \alpha_{2}$ is a one form in the cohomology class $u_{1}$. Moreover, the sublevels associated to $f_{1}$ are the preimages of those associated to $f_{2}$. In particular,

$$
\tilde{\varphi}\left(\left\{f_{1} \leq h\right\}\right) \subset\left\{f_{2} \leq h\right\},
$$

and $\varphi$ induces a homomorphism

$$
\left\lfloor\varphi_{*}\right\rfloor_{h}:\left\lfloor\pi_{1}\left(f_{1}\right)\right\rfloor_{h} \rightarrow\left\lfloor\pi_{1}\left(f_{2}\right)\right\rfloor_{h} .
$$

The collection $\left(\left\lfloor\varphi_{*}\right\rfloor_{h}\right)_{h \in \mathbb{R}}$ forms an inverse system morphism, and in the limit, we obtain a group morphism :

$$
\varphi_{*}: \pi_{1}\left(\tilde{M}_{1}, u_{1}\right) \rightarrow \pi_{1}\left(\tilde{M}_{2}, u_{2}\right) .
$$

Expressing the compatibility of $\varphi_{*}$ with deck transformations requires some more discussion of the latter.

Let $\mathcal{D}_{\tilde{M}_{i}}$ be the deck transformation group of $\tilde{M}_{i}$. Observe that since $\tilde{M}_{2}$ is connected, there a is natural morphism

$$
\mathcal{D}_{\tilde{M}_{1}} \stackrel{\varphi_{\star}}{\longrightarrow} \mathcal{D}_{\tilde{M}_{2}}
$$

Indeed, consider a point $p \in \tilde{M}_{1}$ and a loop $\gamma:[0,1] \rightarrow M_{1}$ based at $\pi(p)$, representing a homotopy class $g_{1} \in \pi_{1}\left(M_{1}\right)$. Let $\tilde{\gamma}$ be the lift of $\gamma$ at $p$, and $\widetilde{\varphi(\gamma)}$ the lift of $\varphi_{*} \gamma$ at $\tilde{\varphi}(p)$. Then $\tilde{\gamma}(1)=g \cdot p$ and $\tilde{\varphi}(\tilde{\gamma}(1))=\left(\widetilde{\varphi_{*} \gamma}\right)(1)$, which means that

$$
\tilde{\varphi}(g \cdot p)=\varphi_{*}(g) \cdot \tilde{\varphi}(p)
$$


In particular, if $g$ and $g^{\prime}$ in $\pi_{1}\left(M_{1}\right)$, have the same action on $\tilde{M}_{1}$, then $\varphi_{*}(g)$ and $\varphi_{*}\left(g^{\prime}\right)$ have the same action at least on a point in $\tilde{M}_{2}$, and since $\tilde{M}_{2}$ is connected, they have the same action on the whole of $\tilde{M}_{2}$.

This means that $\pi_{1}\left(\tilde{M}_{1}\right) \stackrel{\varphi_{*}}{\longrightarrow} \pi_{1}\left(\tilde{M}_{2}\right)$ induces a map $\mathcal{D}_{\tilde{M}_{1}} \stackrel{\varphi_{*}}{\longrightarrow} \mathcal{D}_{\tilde{M}_{2}}$ for which we have :

$$
\forall g \in \mathcal{D}_{\tilde{M}_{1}}, \tilde{\varphi} \circ g=\varphi_{*}(g) \circ \tilde{\varphi}
$$

With this equality we have the following commutative diagram

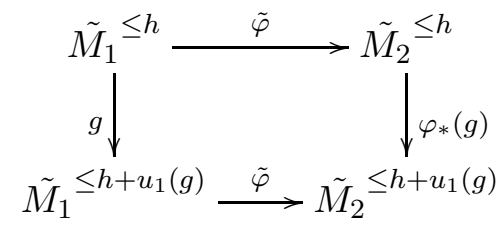

with $\tilde{M}_{2}{ }^{\leq h+u_{1}(g)}=\tilde{M}_{2}{ }^{\leq h+u_{2}\left(\varphi_{*}(g)\right)}$, that induces the commutative diagram on the Novikov $\pi_{1}$.

This ends the proof of point (1) of theorem 1.2. Point (2) follows along the same lines and is no more difficult.

\subsection{Generators and relations.}

2.5.1. Generators up to deck transformations and completion. In general, $\pi_{1}(\tilde{M}, u)$ need not be finitely generated in the usual sense. The object of this section is to define a suitable notion of generators that takes both deck transformations and projective limits into account, for which $\pi_{1}(\tilde{M}, u)$ will be finitely generated.

Given a subset $A \subset \pi_{1}(\tilde{M}, u)=\pi_{1}\left(f_{\alpha}\right)$, consider its orbit $\mathcal{D} \cdot A=\{\tau$. $g, \tau \in \mathcal{D}, g \in A\}$ under all possible deck transformations, and the subgroup $<\mathcal{D}, A>$ generated by $\mathcal{D} \cdot A$ in $\pi_{1}\left(f_{\alpha}\right)$. For each $h \in \mathbb{R}$, the image of this subgroup in $\left\lfloor\pi_{1}\left(f_{\alpha}\right)\right\rfloor_{h}$ defines a group

$$
\lfloor<\mathcal{D}, A>\rfloor_{h}=\zeta_{-}^{h}(<\mathcal{D}, A>) \subset\left\lfloor\pi_{1}\left(f_{\alpha}\right)\right\rfloor_{h}
$$

making the following diagrams commutative

$$
\lfloor<\mathcal{D}, A>\rfloor_{h} \frac{\zeta_{h}^{h^{\prime}}}{\longrightarrow}\lfloor<\mathcal{D}, A>\rfloor_{h^{\prime}} \stackrel{\zeta_{h^{\prime}}^{h^{\prime \prime}}}{\longrightarrow}\lfloor<\mathcal{D}, A>\rfloor_{h^{\prime \prime}}
$$

Definition 2.5. Define the subgroup generated by $A$ up to deck transformations and completion as the group

$$
\left.\overline{<\mathcal{D}, A>}=\lim _{h} L \mathcal{D}, A>\right\rfloor_{h} .
$$

Remark 7. Notice that $\overline{\langle\mathcal{D}, A\rangle}$ is bigger in general than the subgroup $<\mathcal{D}, A>$, since the latter involves only (arbitrary long but) finite products of elements of $\mathcal{D} \cdot A$, while the limit process allows for infinite products.

In the sequel, generated subgroups will always be understood as generated up to deck transforms and completion. 
Proposition 2.6. The group $\overline{\langle\mathcal{D}, A\rangle}$ generated by $A$ in the sense above only depends on the class $u$, and not on the choice of $\alpha$ nor $f_{\alpha}$ used to define it.

Proof. Suppose $\alpha$ and $\beta$ are two 1-forms in the class $u, f_{\alpha}$ and $f_{\beta}$ two primitives of $\alpha$ and $\beta$ on $\tilde{M}$.

Let $K=\left\|f_{\alpha}-f_{\beta}\right\|_{\infty}$. Then the inclusion of sublevels induces, for any level $h \in \mathbb{R}$, the following commutative diagram

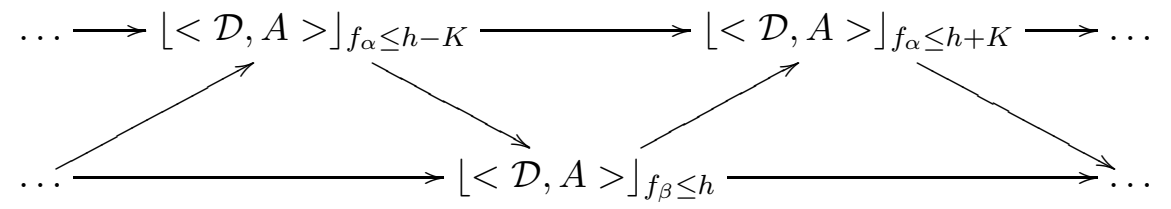

which again induces an isomorphism of projective limits.

Remark 8 . The invariance principle used in this proof shows that the notion of "clean" elements defined in 2.2 does not depend on the choice of $\alpha$ nor $f_{\alpha}$.

2.5.2. Relations up to deck transformations and completion. To define a notion of relation, we need a notion of free group generated by some elements, that still takes the deck transformations and the completion process into account.

Given a finite set $A=\left\{g_{1}, \ldots, g_{k}\right\} \subset \pi_{1}\left(f_{\alpha}\right)$, we consider the product $\mathcal{D} \times A$ and denote by $F_{\mathcal{D} \times A}$ the group freely generated by its elements. Notice it supports an action of the deck transformations group, by multiplication of the letters in a word :

$$
\tau \cdot\left(w_{1} \ldots w_{k}\right)=\left(\tau \cdot w_{1}\right) \ldots\left(\tau \cdot w_{k}\right)
$$

and for each letter $w_{i}=\left(\tau_{i}, g_{i}\right)$

$$
\tau \cdot\left(\tau_{i}, g_{i}\right)=\left(\tau \tau_{i}, g_{i}\right)
$$

The height

$$
h_{f_{\alpha}}(g)=\inf \left\{h \in \mathbb{R}, \zeta_{\infty}^{h}(g)=1 \in\left\lfloor\pi_{1}\left(f_{\alpha}\right)\right\rfloor_{h}\right\}
$$

of elements in $\pi_{1}\left(f_{\alpha}\right)$ extends to $F_{\mathcal{D} \times A}$ at the level of letters by letting

$$
h_{f_{\alpha}}((\tau, g))=u(\tau)+h_{f_{\alpha}}(g) \quad \text { and } \quad h_{f_{\alpha}}\left(w^{-1}\right)=h_{f_{\alpha}}(w)
$$

and at the level of words by letting

$$
h_{f_{\alpha}}\left(w_{1} \ldots w_{k}\right)=\sup _{1 \leq i \leq k}\left\{h_{f_{\alpha}}\left(w_{i}\right)\right\}
$$

Remark 9. In fact, as long as there are finitely many letters, the height of the letters could be fixed arbitrarily. 
Now, for each level $h$ we select the subset

$$
F_{\mathcal{D} \times A}^{\leq h}=\left\{w \in F_{\mathcal{D} \times A}, h_{f_{\alpha}}(w) \leq h\right\},
$$

and consider the group $\left\lfloor F_{\mathcal{D} \times A}\right\rfloor_{h}=F_{\mathcal{D} \times A} / F_{\mathcal{D} \times A}^{\leq h}$.

Observe now that for $h<h^{\prime}<h^{\prime \prime}$, we have compatible morphisms, still denoted by $\zeta$ :

$$
\left\lfloor F_{\mathcal{D} \times A}\right\rfloor_{h} \stackrel{\zeta_{h}^{h^{\prime}}}{\longrightarrow}\left\lfloor F_{\mathcal{D} \times A}\right\rfloor_{h^{\prime}} \stackrel{\zeta_{h^{\prime}}^{h^{\prime \prime}}}{\longrightarrow}\left\lfloor F_{\mathcal{D} \times A}\right\rfloor_{h^{\prime \prime}}
$$

which map all the letters whose height is too small to 1 .

Definition 2.7. Define the group freely generated by $A$ up to deck transformations and completion by

$$
\overline{F_{\mathcal{D} \times A}}={\underset{\varliminf}{h}}_{\lim _{h}}\left\lfloor F_{\mathcal{D} \times A}\right\rfloor_{h} .
$$

The evaluation map sending each letter $g_{i}$ to the element $g_{i} \in \pi_{1}\left(f_{\alpha}\right)$ induces morphisms

$$
\begin{array}{ccc}
\left\lfloor F_{\mathcal{D} \times A}\right\rfloor_{h} & \stackrel{\mathrm{ev}_{h}}{\longrightarrow} & \left\lfloor\pi_{1}\left(f_{\alpha}\right)\right\rfloor_{h+K} \\
\prod_{j=1}^{N}\left(\tau_{j}, g_{i_{j}}\right) & \mapsto & \zeta_{\infty}^{h+K}\left(\prod_{j=1}^{N} \tau_{j} \cdot g_{i_{j}}\right)
\end{array}
$$

where $K$ is a constant, that can be taken to be 1 when the convention (2.4) is used to define the height, but more generally satisfies :

$$
\forall i \in\{1, \ldots, m\}: h_{f_{\alpha}}\left(g_{i}\right)=h \Rightarrow \zeta_{\infty}^{h+K}\left(g_{i}\right)=1 .
$$

Finally, the applications $\mathrm{ev}_{h}$ induce a morphism :

$$
\overline{F_{\mathcal{D} \times A}} \stackrel{\mathrm{ev}}{\longrightarrow} \overline{<\mathcal{D}, A>} \subset \pi_{1}\left(f_{\alpha}\right) .
$$

Definition 2.8. Define the group $\mathcal{R}(A)$ of relations associated to a generating family $A$ of $\pi_{1}\left(f_{\alpha}\right)$ up to DTC as the kernel of

$$
\overline{F_{\mathcal{D} \times A}} \stackrel{\text { ev }}{\rightarrow} \pi_{1}\left(f_{\alpha}\right) .
$$

Remark 10. This kernel is compatible with the filtration, i.e.

$$
\forall w \in \overline{F_{\mathcal{D} \times A}}, w \in \text { ker ev } \Leftrightarrow \forall h \in \mathbb{Z}, \zeta_{\infty}^{h}(w) \in \operatorname{ker}_{h} .
$$

The relations are not finitely generated in general, but there is again a notion of subgroup "normally" generated up to DTC by a collection of elements : given a set $B \subset \mathcal{R}(A)$, define $B^{\prime}=\left\{g b g^{-1}, g \in \overline{F_{\mathcal{D} \times A}}, b \in B\right\}$ and let

$$
{\overline{\langle\mathcal{D}, B\rangle^{*}}}^{*} \overline{\left\langle\mathcal{D}, B^{\prime}>\right.}
$$

be the subgroup generated up to DTC by all the conjugates of elements of $B$.

Given a set $A \subset \pi_{1}\left(f_{\alpha}\right)$ of generators of $\pi_{1}\left(f_{\alpha}\right)$ up to DTC, we let

$$
\rho_{D T C}(A)=\inf \left\{\sharp B, B \subset \mathcal{R}(A) \text { with } \mathcal{R}(A)=\overline{<\mathcal{D}, B>^{*}}\right\} \in \overline{\mathbb{R}}
$$


Definition 2.9. Let

$\mu_{D T C}\left(\pi_{1}\left(f_{\alpha}\right)\right)=\inf \left\{\sharp A, A \subset \pi_{1}\left(f_{\alpha}\right), \pi_{1}\left(f_{\alpha}\right)\right.$ is generated by $A$ up to DTC $\}$

and

$\rho_{D T C}\left(\pi_{1}\left(f_{\alpha}\right)\right)=\inf \left\{\rho_{D T C}(A), A \subset \pi_{1}\left(f_{\alpha}\right), \pi_{1}\left(f_{\alpha}\right)\right.$ is generated by $A$ up to DTC $\}$

denote the minimal number of generators and relations (respectively) of $\pi_{1}\left(f_{\alpha}\right)$.

Remark 11. The invariance argument (see remark 6) shows that the numbers $\mu_{D T C}\left(\pi_{1}\left(f_{\alpha}\right)\right)$ and $\rho_{D T C}\left(\pi_{1}\left(f_{\alpha}\right)\right)$ do not depend on the choices of $\alpha$ and $f_{\alpha}$, but only on $\pi_{1}(\tilde{M}, u)$.

The object of the next section is to prove the following :

Theorem 2.10. The group $\pi_{1}(\tilde{M}, u)$ is finitely generated up to deck transformations and completion, and for all Morse 1-form $\alpha$ in the cohomology class $u$, we have

$$
\sharp\left(\operatorname{Crit}_{1}(\alpha)\right) \geq \mu_{D T C}\left(\pi_{1}\left(f_{\alpha}\right)\right) .
$$

Moreover, the relations are also finitely generated up to deck transformations and completion, and

$$
\sharp\left(\operatorname{Crit}_{2}(\alpha)\right) \geq \rho_{D T C}\left(\pi_{1}\left(f_{\alpha}\right)\right) .
$$

Remark 12. A Morse version of Theorem 2.10 is stated in Sharko1993, Theorem 7.10].

Remark 13. The proof provides a slightly sharper result, where only "clean" generators (see definition 2.2) are taken into account.

\section{Morse theORETiC Interpretation}

3.1. Morse-Novikov steps as generators. Suppose now that $\alpha$ is a Morse 1 -form in the cohomology class $u \in H^{1}(M, \mathbb{R})($ with $u \neq 0)$.

Pick also

- a metric $<,>$ on $M$ such that the pair $(\alpha,<,>)$ is Morse-Smale,

- a primitive $f_{\alpha}$ of $\alpha$ on $\tilde{M}$,

- a preferred lift $\underline{\tilde{c}}$ to $\tilde{M}$ of each $\underline{c} \in \operatorname{Crit}(\alpha)$; this allows for the identification

$$
\operatorname{Crit}\left(f_{\alpha}\right)=\{\tau \cdot \underline{\tilde{c}}, \tau \in \mathcal{D}, \underline{c} \in \operatorname{Crit}(\alpha)\} .
$$

- an arbitrary orientation on the unstable manifold of each $c \in \operatorname{Crit}(\alpha)$; this picks a preferred orientation on the unstable manifolds of all the critical points of $f_{\alpha}$.

For convenience, we suppose $u \neq 0$. This allows us to pick, for each index 0 critical point $\underline{x} \in \operatorname{Crit}_{0}(\alpha)$, an arbitrary path $\gamma_{\underline{\tilde{x}}}:[0,+\infty) \rightarrow \tilde{M}$ such that

- $\gamma_{\underline{\tilde{x}}}(0)=\underline{\tilde{x}}$

- $\lim _{t \rightarrow+\infty} f_{\alpha}\left(\gamma_{\underline{\tilde{x}}}(t)\right)=-\infty$. 
In fact, we can pick a loop $l$ in $M$ based at $\underline{x}$ such that $u(l)<0$, and lift the iterations of $l$ to $\tilde{M}$.

Notice that, letting

$$
\gamma_{\tau \cdot \underline{\tilde{x}}}=\tau \cdot \gamma_{\underline{\tilde{x}}}
$$

automatically selects, for each index 0 critical point $x$ of $f_{\alpha}$, a preferred path $\gamma_{x}$ in $\tilde{M}$ from $x$ to $-\infty$.

There are finitely many index 0 critical points for $\alpha$, so there is an upper bound $\kappa$ on the height these paths can reach above their starting point :

$$
\exists \kappa \in \mathbb{R}, \forall x \in \operatorname{Crit}_{0}\left(f_{\alpha}\right), \quad \gamma_{x} \subset \tilde{M}^{\leq f_{\alpha}(x)+\kappa}
$$

To each index 1 critical point $y$ of $f_{\alpha}$, the unstable manifold of $y$, endowed with its preferred orientation, defines a path $\gamma:(-\infty,+\infty) \rightarrow \tilde{M}$ such that near both ends, we have the alternative

$$
\lim _{t \rightarrow-\infty} f_{\alpha}(\gamma(t))=-\infty \text { or } \lim _{t \rightarrow-\infty} f_{\alpha}(\gamma(t))=x_{-} \in \operatorname{Crit}_{0}\left(f_{\alpha}\right)
$$

and

$$
\lim _{t \rightarrow+\infty} f_{\alpha}(\gamma(t))=-\infty \text { or } \lim _{t \rightarrow+\infty} f_{\alpha}(\gamma(t))=x_{+} \in \operatorname{Crit}_{0}\left(f_{\alpha}\right) .
$$

In the former case, the corresponding flow line is said to be infinite, or unbounded. In the latter case, it is said to be bounded, and a suitable reparameterisation (using the value of $f_{\alpha}$ as the new parameter for instance) and concatenation with the path $\gamma_{x_{ \pm}}$extends the flow line into a path going to $-\infty$. Doing this continuation at one or both ends if needed, we obtain in all cases a continuous path

$$
\gamma_{y}:(-\infty,+\infty) \rightarrow \tilde{M} \text { with } \lim _{t \rightarrow \pm \infty} f_{\alpha}\left(\gamma_{y}(t)\right)=-\infty
$$

which we call the Morse-Novikov step associated to $y$.
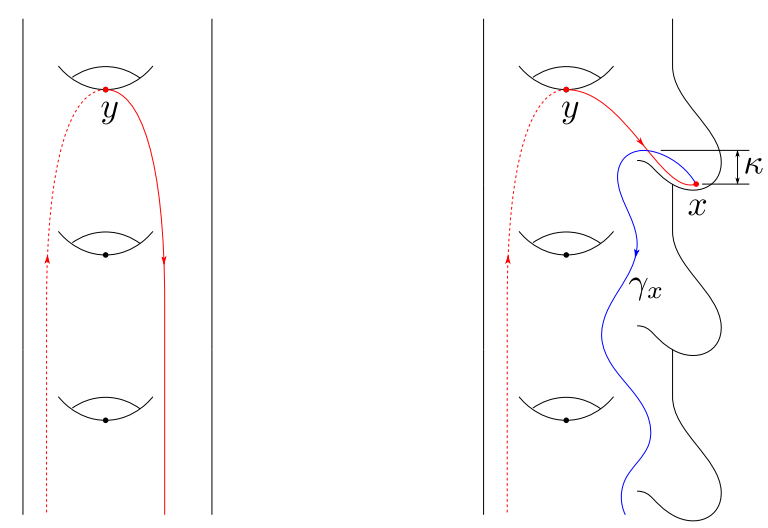

Figure 2. Morse-Novikov steps. Whenever a flow line rooted at $y$ ends at an index 0 critical point $x$, it is extended with the path $\gamma_{x}$. 
Definition 3.1. Let $\mathfrak{G}_{\alpha}$ be the collection of Morse-Novikov steps associated to the index 1 critical points of $\alpha$ :

$$
\mathfrak{G}_{\alpha}=\left\{\gamma_{\underline{y}}, \underline{y} \in \operatorname{Crit}_{1}(\alpha)\right\} .
$$

Each such path $\gamma_{\tilde{y}}$ defines a class $g_{\tilde{y}}=\left[\gamma_{\tilde{y}}\right] \in \pi_{1}\left(f_{\alpha}\right)$, and $\mathfrak{G}_{\alpha}$ will often be implicitly and abusively considered as a finite subset in $\pi_{1}\left(f_{\alpha}\right)$, by considering the collection $\left\{g_{\underline{y}}\right\}$ instead of $\left\{\gamma_{\underline{y}}\right\}$.

Remark 14. The Morse-Novikov steps are "clean" elements in the sense of definition 2.2 ,

Proposition 3.2. The collection $\mathfrak{G}_{\alpha}$ is a finitely generating family (up to deck transformations and completion) for $\pi_{1}(\tilde{M}, u)$ :

$$
\pi_{1}(\tilde{M}, u)=\overline{\left\langle\mathcal{D}, \mathfrak{G}_{\alpha}>\right.}=\overline{\left\langle\mathcal{D},\left\{g_{\underline{\tilde{y}}}, \underline{y} \in \operatorname{Crit}_{1}\left(f_{\alpha}\right)\right\}>\right.} .
$$

A straightforward corollary of this proposition is the following :

Corollary 3.3. For all Morse 1-form $\alpha$ in the class $u$ we have

$$
\sharp \operatorname{Crit}_{1}(\alpha) \geq \mu_{D T C}\left(\pi_{1}(\tilde{M}, u)\right) \text {. }
$$

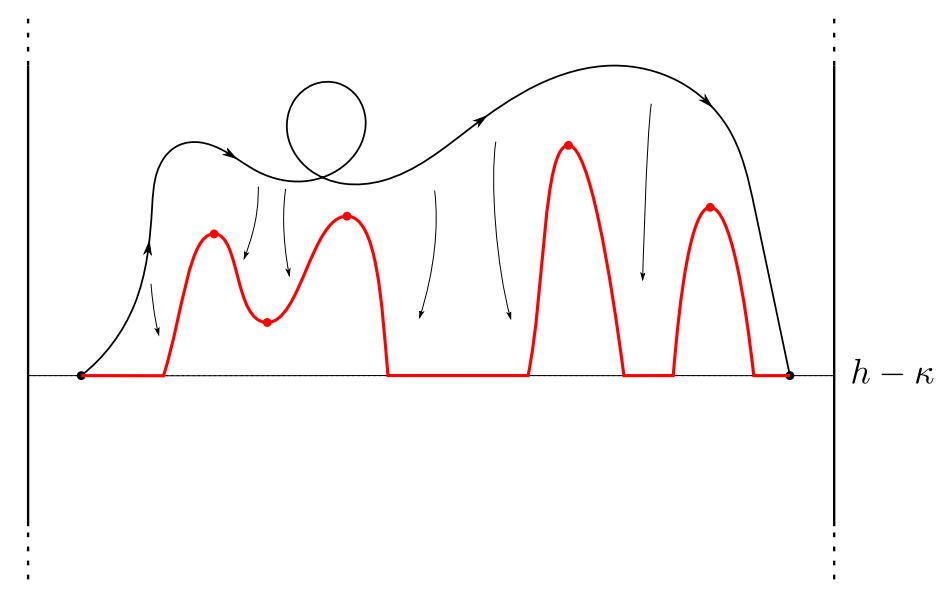

FIGURE 3. Starting from an arbitrary loop, flow it down...

Proof. Fix some regular level $h$ of $f_{\alpha}$. Let $g \in \pi_{1}\left(f_{\alpha}\right)$, and $\gamma:[0,1] \rightarrow$ $\lfloor\tilde{M}\rfloor_{h-\kappa}$ be a path representing $\zeta^{h-\kappa}(g)$ in $\left\lfloor\pi_{1}\left(f_{\alpha}\right)\right\rfloor_{h-\kappa}$ (recall $\kappa$ is the constant defined in (3.1)).

The path $\gamma$ defines a finite collection of paths $\left(\gamma_{1}, \ldots, \gamma_{k}\right)$ from $[0,1]$ to $\tilde{M}$ with ends on $\left\{f_{\alpha}=h-\kappa\right\}$.

Pushing a component $\gamma_{i}$ down by the gradient flow of $-f_{\alpha}$ moves it either below level $h$ or onto unstable manifolds of index 1 critical points. More precisely, there is a time $T$ after which the curve is contained in the union of the sublevel $\tilde{M}^{\leq h}$ and small neighborhoods of the unstable manifolds of 


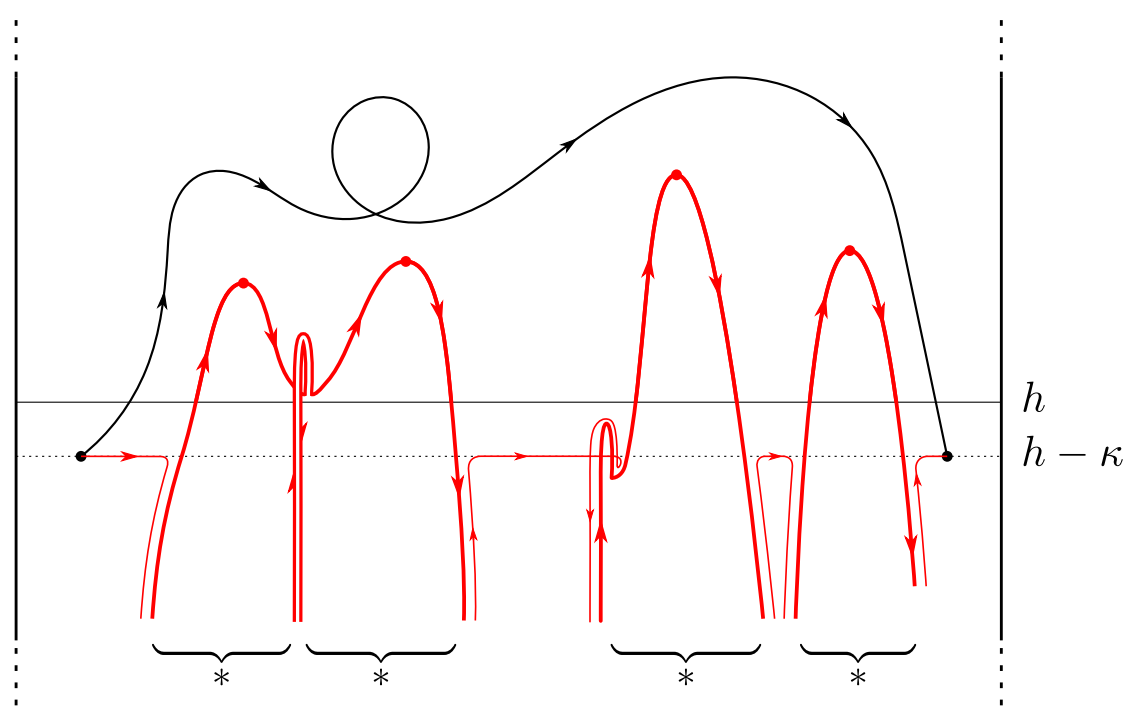

FiguRE 4. ... and complete it to get a sequence of MorseNovikov steps. The paths marked with a $(*)$ are MorseNovikov steps, the other are contained in $\tilde{M}^{\leq h}$.

some index 1 critical points. A classical retraction argument then turns it into the concatenation $\gamma_{i}^{\prime}$ of paths that are (see figure 3).

(1) either a path in $\tilde{M}^{\leq h}$,

(2) or a piece of unstable manifold of $y \in \operatorname{Crit}_{1}\left(f_{\alpha}\right)$ whose ends are either in $\tilde{M}^{\leq h}$ or some index 0 critical point $x \in \operatorname{Crit}_{0}\left(f_{\alpha}\right)$ with $f_{\alpha}(x)>h-\kappa$.

Notice that since the initial path $\gamma_{i}$ starts and ends in $\tilde{M}^{\leq h}$, so does $\gamma_{i}^{\prime}$.

To turn $\gamma_{i}^{\prime}$ into a product of Morse-Novikov steps, we finally apply the following modifications at the bottom of each piece of flow line used in $\gamma_{i}^{\prime}$ :

(1) if the flow line is unbounded, we insert a two way trip down to $-\infty$ along the flow line,

(2) if the flow line is bounded, we insert a two way trip along the remaining part of the flow line if required, and along the preferred path $\gamma_{x}$ associated to this critical point down to $-\infty$.

This operation completes each arc of unstable manifold in $\gamma_{i}^{\prime}$ into the associated Morse-Novikov step.

Each piece $\eta$ of $\gamma_{i}^{\prime}$ that is contained in $\tilde{M}^{\leq h-\kappa}$ is now completed

- either by a flow line that goes to $-\infty$, which is necessarily also contained in $\tilde{M}^{\leq h-\kappa}$,

- or a piece of flow line down to some critical point $x \in \operatorname{Crit}_{0}\left(f_{\alpha}\right)$, followed by the path $\gamma_{x}$. Since $x$ has to be on a lower level than the 
start or end of $\eta$, we have $f_{\alpha}(x) \leq h-\kappa$, and then by definition of $\kappa, \gamma_{x}$ cannot go higher than the level $(h-\kappa)+\kappa=h$.

As a consequence we obtain a sequence of paths from and to $-\infty$, such that

(1) each path is either a Morse-Novikov step or lies in $\tilde{M}^{\leq h}$,

(2) in $\lfloor\tilde{M}\rfloor_{h}$, the concatenation of these paths is homotopic to $\gamma_{i}$.

Finally, $\gamma_{i}^{\prime}$ is homotopic in $\lfloor\tilde{M}\rfloor_{h}$ the projection of a product of MorseNovikov steps :

$$
\gamma_{i}^{\prime} \sim \gamma_{y_{1}}^{ \pm 1} \cdots \gamma_{y_{k}}^{ \pm 1} \quad \text { in } \quad\lfloor\tilde{M}\rfloor_{h}
$$

In particular, we obtain that the class $g$ we started with satisfies

$$
\zeta^{h}(g) \in\left\lfloor<\mathcal{D}, \mathfrak{G}_{\alpha}>\right\rfloor_{h} .
$$

This proves that $\forall h \in \mathbb{R},\left\lfloor\pi_{1}\left(f_{\alpha}\right)\right\rfloor_{h} \subset\left\lfloor<\mathcal{D}, \mathfrak{G}_{\alpha}>\right\rfloor_{h}$ and hence

$$
\pi_{1}\left(f_{\alpha}\right)=\overline{<\mathcal{D}, \mathfrak{G}_{\alpha}>} \text {. }
$$

3.2. Morse-Novikov relations. Similarly, consider an index 2 critical point $z \in \mathrm{Crit}_{2}\left(f_{\alpha}\right)$.

Given a level $h$ with $h<f_{\alpha}(z)$, the set $W^{u}(z) \cap\left\{f_{\alpha}>h\right\}$ is a topological disc (notice that once a trajectory enters the sublevel $\left\{f_{\alpha} \leq h\right\}$, it will never exit it anymore). Moreover, starting with a small circle around $z$ inside its unstable manifold, and pushing it down by the flow using the technique described earlier, we obtain a loop $\rho_{z, h}$ whose projection in $\lfloor\tilde{M}\rfloor_{h}$ is that of a product of Morse-Novikov steps :

$$
\rho_{z, h} \sim \gamma_{y_{1}}^{ \pm 1} \cdots \gamma_{y_{k}}^{ \pm 1} \quad \text { in } \quad\lfloor\tilde{M}\rfloor_{h} .
$$

In particular, considering the group $F=\overline{F_{\mathcal{D} \times \operatorname{Crit}_{1}(\alpha)}}$ freely generated by the index 1 critical points of $\alpha$ (up to deck transformations and completion), the sequence $\left(y_{1}^{ \pm 1}, \ldots, y_{k}^{ \pm 1}\right)$, after removal of the eventual critical points $y$ such that $h-\kappa<f_{\alpha}(y) \leq h$, defines a word

$$
w_{z, h}=y_{1}^{ \pm 1} \ldots y_{k}^{ \pm 1} \in\lfloor F\rfloor_{h} .
$$

Moreover, these words are compatible with inclusion of sublevels, namely, for $h<h^{\prime}$, we have :

$$
\zeta_{h}^{h^{\prime}}\left(w_{z, h}\right)=w_{z, h^{\prime}}
$$

As a consequence, the words $\left(w_{z, h}\right)_{h}$ define a class $w_{z}$ in $F$.

Definition 3.4. Define the relation associated to a critical point $\underline{z} \in \mathrm{Crit}_{2}(\alpha)$ as the word $w_{\underline{z}}$, and let

$$
\Re_{\alpha}=\left\{w_{\underline{z}}, \underline{z} \in \operatorname{Crit}_{2}(\alpha)\right\} .
$$


Proposition 3.5. The relations associated to the generating family given by the index 1 critical points of $\alpha$ is normally spanned by the relations associated to the index 2 critical points :

$$
\mathcal{R}\left(\mathfrak{G}_{\alpha}\right)=\overline{\left\langle\mathcal{D}, \mathfrak{R}_{\alpha}>^{*}\right.}
$$

A straightforward corollary of this proposition is the following :

Corollary 3.6. For all Morse 1-form $\alpha$ in the class $u$ we have

$$
\sharp \operatorname{Crit}_{2}(\alpha) \geq \rho_{D T C}\left(\pi_{1}(\tilde{M}, u)\right) .
$$

Proof. By construction, the elements in $\overline{\left\langle\mathcal{D}, \mathfrak{R}_{\alpha}\right\rangle^{*}}$ are indeed relations. On the other hand, let $w \in F=\overline{F_{\mathcal{D} \times \mathfrak{G}_{\alpha}}}$ and suppose $w$ evaluates to 1 in all $\left\lfloor\pi_{1}\left(f_{\alpha}\right)\right\rfloor_{h}$ (via the map defined in (2.6) ).

Fix a level $h$, and consider $w_{h}=\zeta_{\infty}^{h}(w) \in\lfloor F\rfloor_{h}$ and $\gamma_{h}$ its evaluation in $\lfloor\tilde{M}\rfloor_{h}$. Since $\left[\gamma_{h}\right]=1$ in $\left\lfloor\pi_{1}\left(f_{\alpha}\right)\right\rfloor_{h}$, there is a disc $\delta: D^{2} \rightarrow\lfloor\tilde{M}\rfloor_{h}$ with boundary on $\gamma_{h}$.

Pushing this disc down by the flow, we obtain a new disc $\delta^{\prime}: D^{2} \rightarrow\lfloor\tilde{M}\rfloor_{h}$, that has the same boundary (since it did already consist of flow lines) and splits as a union of unstable manifolds of index 2 critical points (seen in $\lfloor\tilde{M}\rfloor_{h}$ ), and regions where it evaluates to the base point $\left[\tilde{M}^{\leq h}\right]$.

In particular, we obtain that $\gamma_{h}$ can be written in $\lfloor\tilde{M}\rfloor_{h}$ as a product of conjugates of the relations associated to index 2 critical points.

\section{HuREWICZ HOMOMORPHISM}

In this section we construct a Hurewicz morphism in the Novikov setting. We refer to [Sikorav1987, Farber2004] for a definition of the Novikov homology $H N_{*}(\tilde{M}, u)$ associated to a cohomology class $u \in H^{1}(M, \mathbb{R})$ and an associated integration cover $\tilde{M}$. The Novikov homology associated to the minimal integration cover will be denoted as $H N_{*}(M, u)$, and the one associated to the universal cover by $H N_{*}\left(M, u ; \mathbb{Z}\left[\pi_{1}(M)\right]\right)$.

4.1. Commutators and projective limits. To describe the kernel of the Hurewicz morphism in the Novikov setting, we first need to discuss commutators in relation to projective limits. Let $\left(G_{h}\right)_{h}$ be a projective system of groups and $G=\lim G_{h}$. Then both the commutators $\left(\left[G_{h}, G_{h}\right]\right)_{h}$ and the abelianizations $\left(\overleftarrow{G_{h}^{\mathrm{ab}}}\right)_{h}=\left(G_{h} /\left[G_{h}, G_{h}\right]\right)_{h}$ form two other projective systems. The projective limit of the commutator subgroup $\lim _{\longleftarrow}\left[G_{h}, G_{h}\right]$ form a normal subgroup of $G$ that contains the usual commutator subgroup $[G, G]$ of $G$. We refer to it as the pro-commutator subgroup.

Recall that a projective system $\left(G_{h}\right)_{h}$ with maps $G_{h} \stackrel{\zeta_{h}^{h^{\prime}}}{\longrightarrow} G_{h^{\prime}}$ is said to satisfy the Mittag-Leffler condition if there is a constant $K$ such that, for each fixed level $h_{0}$, the maps $\zeta_{h}^{h_{0}}: G_{h} \rightarrow G_{h_{0}}$ have the same range in $G_{h_{0}}$ for all $h \leq h_{0}-K$. 
Proposition 4.1. If the projective system $\left(G_{h}\right)_{h}$ satisfies the Mittag-Leffler condition, then

$$
G / \lim _{\longleftarrow}\left[G_{h}, G_{h}\right] \simeq \lim \left(G_{h} /\left[G_{h}, G_{h}\right]\right) .
$$

This group will be denoted as $G \frac{\mathrm{ab}}{}$ and referred to as the pro-abelianization of $G$ (although it depends on the projective system $\left(G_{h}\right)_{h}$ and not on $G$ only).

Notice that since $[G, G]$ is a subgroup of $\lim _{\longleftarrow}\left[G_{h}, G_{h}\right]$, there is a projection

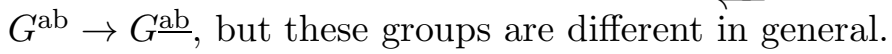

Proof. At each level, we have the following exact sequence

$$
0 \rightarrow\left[G_{h}, G_{h}\right] \rightarrow G_{h} \rightarrow G_{h} /\left[G_{h}, G_{h}\right] \rightarrow 0,
$$

which form in fact an exact sequence of projective systems.

Observe now that if $\left(G_{h}\right)_{h}$ satisfies the Mittag-Leffler condition, so does $\left(\left[G_{h}, G_{h}\right]\right)_{h}$. As a consequence, we have an exact sequence among the projective limits :

$$
0 \rightarrow \lim _{\longleftarrow}\left[G_{h}, G_{h}\right] \rightarrow G \rightarrow \lim _{\longleftarrow}\left(G_{h} /\left[G_{h}, G_{h}\right]\right) \rightarrow 0,
$$

which shows that $G / \lim _{\longleftarrow}\left[G_{h}, G_{h}\right]$ is isomorphic to $\lim _{\longleftarrow}\left(G_{h} /\left[G_{h}, G_{h}\right]\right)$.

Lemma 4.2. The Novikov fundamental group satisfies the Mittag-Leffler condition, namely, if $\alpha$ is a 1-form in the class $u$, and $f_{\alpha}$ a primitive of $\alpha$ on $\tilde{M}$, then there exist a constant $\kappa$ such that, for all level $h \in \mathbb{R}$ the maps

$$
\left\lfloor\pi_{1}\left(f_{\alpha}\right)\right\rfloor_{h^{\prime}} \stackrel{\zeta_{h^{\prime}}^{h}}{\longrightarrow}\left\lfloor\pi_{1}\left(f_{\alpha}\right)\right\rfloor_{h}
$$

have all the same range provided $h^{\prime}<h-\kappa$.

Remark 15. The Mittag-Leffler condition is a condition on projective systems, and does not make sense for a single group. However, as observed in remark 6, the Novikov fundamental group comes with a preferred collection of essentially equivalent projective systems, and the statement claims they do all satisfy the Mittag-Leffler condition.

Proof. The invariance argument shows that this statement does not depend on the choice of the one form $\alpha$ nor on the primitive $f_{\alpha}$.

More explicitly, let $f_{\alpha}$ and $f_{\beta}$ be two primitives of two 1-forms $\alpha$ and $\beta$ in the cohomology class $u$, and suppose $\pi_{1}\left(f_{\alpha}\right)$ satisfies the Mittag-Leffler condition for a constant $\kappa$. Recall that $K=\left\|f_{\alpha}-f_{\beta}\right\|_{\infty}<+\infty$. Then the following commutative diagram

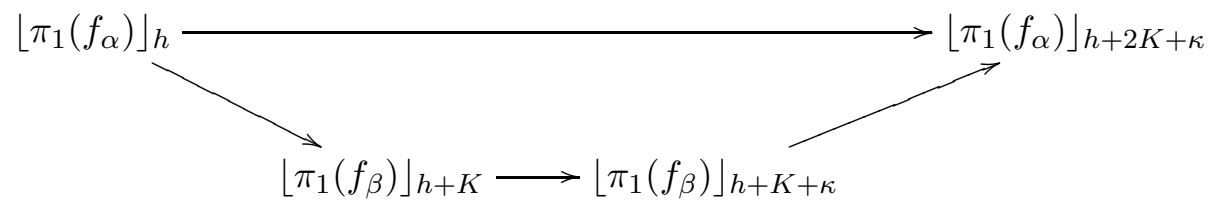

shows that $\pi_{1}\left(f_{\beta}\right)$ satisfies the Mittag-Leffler condition for the constant $2 K+$ $\kappa$. 
So we can suppose without loss of generality that $\alpha$ is Morse, and consider the constant $\kappa$ already defined in (3.1) : for each index 0 critical point $x$ of $f_{\alpha}$, there is a path $\gamma_{x}$ that starts at $x$ and goes down to $-\infty$ (i.e. $\left.\lim _{t \rightarrow+\infty} f_{\alpha}\left(\gamma_{x}(t)\right)=-\infty\right)$, and does not go higher than $f_{\alpha}(x)+\kappa$.

In particular, if $\gamma:[0,1] \rightarrow \tilde{M}$ is a path that starts and ends below a level $h-\kappa$ (i.e. $f_{\alpha}(\gamma(0)) \leq h$ and $f_{\alpha}(\gamma(1)) \leq h$ ) with generic ends, we can flow its ends down to either $-\infty$ or an index 0 critical point $x$, in which case we extend the resulting path by $\gamma_{x}$. Through this process, $\gamma$ is extended as a path $\bar{\gamma}: \mathbb{R} \rightarrow \tilde{M}$ such that :

(1) $\lim _{t \rightarrow \pm \infty} f_{\alpha}(\bar{\gamma}(t))=-\infty$

(2) $\bar{\gamma}$ and $\gamma$ are the same above level $h$, more precisely $\bar{\gamma}: \mathbb{R} \rightarrow\lfloor\tilde{M}\rfloor_{h}$ and $\gamma: \mathbb{R} \rightarrow\lfloor\tilde{M}\rfloor_{h}$ (where $\gamma$ is extended away from $[0,1]$ as the constant map to the base point) are the same maps. of

In particular, this proves that if an element $[\gamma] \in\left\lfloor\pi_{1}\left(f_{\alpha}\right)\right\rfloor_{h}$ is in the image

$$
\left\lfloor\pi_{1}\left(f_{\alpha}\right)\right\rfloor_{h-\kappa} \stackrel{\zeta_{h-\kappa}^{h}}{\longrightarrow},\left\lfloor\pi_{1}\left(f_{\alpha}\right)\right\rfloor_{h}
$$

it is also in the image of

$$
\left\lfloor\pi_{1}\left(f_{\alpha}\right)\right\rfloor_{h^{\prime}} \stackrel{\zeta_{h^{\prime}}^{h}}{\longrightarrow},\left\lfloor\pi_{1}\left(f_{\alpha}\right)\right\rfloor_{h}
$$

for all $h^{\prime} \leq h-\kappa$.

Moreover, the invariance argument also shows the pro-commutator subgroup and the pro-abelianization group do not depend on the choice of $\alpha$ or $f_{\alpha}$, so that we end up with a well defined group

$$
\pi_{1}(\tilde{M}, u)^{\underline{\mathrm{ab}}}=\pi_{1}\left(f_{\alpha}\right)^{\mathrm{ab}}=\lim _{\longleftarrow}\left(\left\lfloor\pi_{1}\left(f_{\alpha}\right)\right\rfloor_{h}\right)^{\mathrm{ab}} .
$$

4.2. Hurewicz morphism. The object of this section is to establish the following theorems.

In the case where $\tilde{M}$ is the minimal integration cover, we have the following result :

Theorem 4.3. Let $u \in H^{1}(M, \mathbb{R})$ be a cohomology class, and $\tilde{M}$ be its minimal integration cover.

Then there is a surjective homomorphism

$$
\mathfrak{h}: \pi_{1}(\tilde{M}, u) \rightarrow H N_{1}(M, u ; \mathbb{Z}) .
$$

The kernel of $\mathfrak{h}$ consists of the pro-commutator subgroup of $\pi_{1}(\tilde{M}, u)$. In particular, $\mathfrak{h}$ induces an isomorphism

$$
\mathfrak{h}: \pi_{1}(\tilde{M}, u)^{\underline{\mathrm{ab}}} \simeq H N_{1}(M, u ; \mathbb{Z}) .
$$

For more general integration covers, we have the slightly weaker result :

Theorem 4.4. Let $u \in H^{1}(M, \mathbb{R})$ be a cohomology class, and $\tilde{M}$ be one of its integration cover. 
Then there is a surjective homomorphism

$$
\mathfrak{h}: \pi_{1}(\tilde{M}, u) \rightarrow \underset{h}{\lim _{h}} H_{1}\left(\tilde{M}, \tilde{M}^{\leq h}\right) .
$$

The kernel of $\mathfrak{h}$ consists of the pro-commutator subgroup of $\pi_{1}(\tilde{M}, u)$. In particular, $\mathfrak{h}$ induces an isomorphism

$$
\mathfrak{h}: \pi_{1}(\tilde{M}, u)^{\underline{\mathrm{ab}}} \stackrel{\simeq}{\underset{h}{\lim }} H_{1}\left(\tilde{M}, \tilde{M}^{\leq h}\right) .
$$

Theorem 4.3 follows from theorem 4.4 and an improved description of $\lim _{h} H_{1}\left(\tilde{M}, \tilde{M}^{\leq h}\right)$ in terms of Novikov homology in the particular cases when $\tilde{M}$ is the minimal integration cover.

Proof of theorem 4.3 from theorem 4.4. Recall that the Novikov complex can be interpreted as a projective limit of relative complexes [Sikorav1987] :

$$
C N_{*}(M, u)=\lim _{h} C_{*}\left(\tilde{M}, \tilde{M}^{\leq h}\right)
$$

In [Sikorav1987], J.-C. Sikorav shows that the comparison of the homology of the projective limit and the projective limit of the homologies gives rise to the following short exact sequence

$$
0 \rightarrow \lim ^{1} H_{*+1}\left(\tilde{M}, \tilde{M}^{\leq h}\right) \rightarrow H N_{*}(\tilde{M}, u) \rightarrow{\underset{\leftarrow}{h}}_{\lim _{*}} H_{*}\left(\tilde{M}, \tilde{M}^{\leq h}\right) \rightarrow 0 .
$$

In the particular case where $\tilde{M}$ is the minimal integration cover of $u$, the deck transformation group $\mathcal{D}$ is isomorphic to $\mathbb{Z}^{r}$ for some $r>0$, and M. Usher shows in [Usher2008, Theorem 1.3, p. 1583] that a Novikov chain $c$ that is a boundary of a Novikov chain $d$, is the boundary of a chain $d^{\prime}$ that goes no higher than the maximal height of $c$ plus some constant $M$.

This implies that $H_{*}\left(\tilde{M}, \tilde{M}^{\leq h}\right)$ satisfies the Mittag-Leffler condition, and hence that the $\lim ^{1}$ term in (4.1) vanishes. This ends the proof of theorem 4.3 assuming theorem 4.4 .

Proof of theorem 4.4. Pick a 1-form $\alpha$ in the class $u$ and a primitive $f_{\alpha}$ of $\alpha$ on $\tilde{M}$. Observe that at each regular level $h \in \mathbb{R}$, we have a Hurewicz morphism

$$
\lfloor\mathfrak{h}\rfloor_{h}:\left\lfloor\pi_{1}\left(f_{\alpha}\right)\right\rfloor_{h} \rightarrow H_{1}\left(\lfloor\tilde{M}\rfloor_{h}\right)=H_{1}\left(\tilde{M}, \tilde{M}^{\leq h}\right) .
$$

$\left(H_{1}(X ; A) \simeq H_{1}(X / A)\right.$ holds in general when $A$ is a deformation retract of a neighborhood of $A$ in $X$; when $h$ is a regular level, this is the case for $A=\tilde{M}^{\leq h}$ in $X=\tilde{M}$, since every point has a neighborhood that is either contained in $A$ or pushed back in $A$ by the flow).

Moreover, this sequence of morphisms $\lfloor\mathfrak{h}\rfloor_{h}$ are compatible with the morphisms associated to increasing levels $h<h^{\prime}$. In other words, the following 
diagram

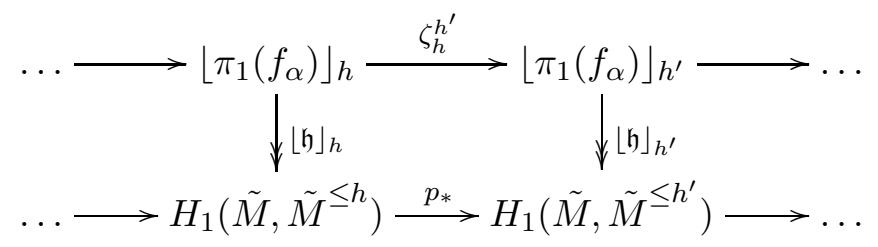

(where $p_{*}$ is the map induced by the projection $\lfloor\tilde{M}\rfloor_{h} \stackrel{p}{\rightarrow}\lfloor\tilde{M}\rfloor_{h^{\prime}}$ ) is commutative. In particular, the sequence of maps $\left(\lfloor\mathfrak{h}\rfloor_{h}\right)_{h}$ defines a morphism

$$
\pi_{1}\left(f_{\alpha}\right) \stackrel{\mathfrak{h}}{\rightarrow} \lim _{\longleftarrow} H_{1}\left(\tilde{M}, \tilde{M}^{\leq h}\right) .
$$

Moreover, at each level, we have the exact sequence

$$
0 \rightarrow\left[\left\lfloor\pi_{1}\left(f_{\alpha}\right)\right\rfloor_{h},\left\lfloor\pi_{1}\left(f_{\alpha}\right)\right\rfloor_{h}\right] \rightarrow\left\lfloor\pi_{1}\left(f_{\alpha}\right)\right\rfloor_{h} \stackrel{\lfloor\mathfrak{h}\rfloor_{h}}{\longrightarrow} H_{1}\left(\tilde{M}, \tilde{M}^{\leq h}\right) \rightarrow 0 .
$$

This defines in fact an exact sequence of projective systems, and since the first term satisfies the Mittag-Leffler condition, we obtain an exact sequence for the projective limits (see [Massey1978, Theorem A.14]). In particular, we obtain that $\mathfrak{h}$ induces an isomorphism

$$
\pi_{1}\left(f_{\alpha}\right) \stackrel{\text { ab }}{\sim} \underset{\mathfrak{h}}{\sim} \lim _{\longleftarrow} H_{1}\left(\tilde{M}, \tilde{M}^{\leq h}\right) .
$$

\section{EXAmples}

In this section we focus on manifolds of the form

$$
M=\mathbb{T}^{n} \sharp X
$$

obtained as the connected sum of a torus and some closed manifold $X$ of dimension $n \geq 4$. For convenience we let $G=\pi_{1}(X)$.

The main example we have in mind is the case where $X=S \times \mathbb{S}^{n-3}$, where $S$ is the Poincaré sphere, in which case we have :

$$
G=\pi_{1}(S)=<a, b \mid a^{5}=b^{3}, a^{5}=(a b)^{2}>,
$$

but other cases will also be considered, and the discussion below holds for general $X$.

Let $\mathbb{T}^{n} \stackrel{\theta}{\rightarrow} \mathbb{S}^{1}$ be the first coordinate on the torus, and consider the cohomology class

$$
u=\pi^{\star} d \theta
$$

where $\pi: M \rightarrow \mathbb{T}^{n}$ is a projection mapping $X$ to a point.

The minimal integration cover of $u$ is then the manifold

$$
\left(\mathbb{R} \times \mathbb{T}^{n-1}\right) \underset{\mathbb{Z}}{\sharp} X
$$

obtained by performing the connected sum of the cylinder with a fresh copy of $X$ at each lift of the point where the connected sum took place on the torus. 
5.1. Basic recollection of Novikov homology for $\mathbb{T}^{n} \sharp X$. We denote by $H N_{\star}(M, u)$ the Novikov homology associated to this covering of $M$. Algebraically, this comes down to considering the group $\mathcal{D}=\pi_{1}(M) / \operatorname{ker} u \simeq \mathbb{Z}$ as defining a local coefficients system, and use coefficients in the Novikov completion $\Lambda=\mathbb{Z}((t))$ of the group ring $\mathbb{Z}[\mathcal{D}]=\mathbb{Z}\left[t, t^{-1}\right]$. Namely,

$$
\Lambda=\left\{\sum_{-N}^{+\infty} a_{n} t^{n}, a_{n} \in \mathbb{Z}\right\}
$$

is the set of Laurent power series over $\mathbb{Z}$, and we consider the complex

$$
C N_{*}(M, u)=\Lambda \otimes_{\mathbb{Z}\left[t, t^{-1}\right]} C_{*}\left(M, \mathbb{Z}\left[t, t^{-1}\right]\right)
$$

where $C_{*}\left(M, \mathbb{Z}\left[t, t^{-1}\right]\right)$ is (for instance) the simplicial chain complex of $M$ with local coefficients in $\mathbb{Z}[\mathcal{D}]=\mathbb{Z}\left[t, t^{-1}\right]$.

Finally, the Novikov homology $H N_{*}(M, u)$ is the homology of $C N_{*}(M, u)$.

Proposition 5.1. With the above notations, we have $H N_{0}(M, u)=0$, and for $i=1,2$ :

$$
H N_{i}(M, u)=H_{i}(X) \underset{\mathbb{Z}}{\otimes} \Lambda .
$$

Proof. Vanishing of $H N_{0}(M, u)$ whenever $u \neq 0$ is a classical and general feature of Novikov homology.

To compute $H N_{i}(M, u)(i=1,2)$ we use the universal coefficient formula and the flatness of the Novikov ring $\mathbb{Z}((t))$ as a $\mathbb{Z}\left[t, t^{-1}\right]$-module Pajitnov2006 Theorem 1.8, p. 339], cf. [LO1995, Appendix C]

$$
H N_{i}(M, u)=H_{i}(\tilde{M}) \otimes_{\mathbb{Z}\left[t, t^{-1}\right]} \mathbb{Z}((t)) .
$$

To compute $H_{i}(\tilde{M})$, we split $\tilde{M}$ as the union of

- $A=\left(\mathbb{R} \times \mathbb{T}^{n-1}\right) \backslash\left\{p_{k}, k \in \mathbb{Z}\right\}$ where the $p_{k}$ are the lifts of the point where the connected sum was performed,

- $B=\sqcup_{k \in \mathbb{Z}}(X \backslash\{p\})_{k}$ the disjoint union of $\mathbb{Z}$ punctured copies of $X$.

The overlapping region $A \cap B$ is the disjoint union of $\mathbb{Z}$ copies of $\mathbb{S}^{n-1} \times$ $[0,1]$, which has vanishing homology groups in degrees 1 and 2 provided $n \geq 4$.

By the Mayer-Vietoris sequence theorem we have for $i=1,2$

$$
H_{i}(\tilde{M})=H_{i}(X)^{\mathbb{Z}} \oplus H_{i}\left(\mathbb{R} \times \mathbb{T}^{n-1}\right) .
$$

Since $\mathcal{D}=\mathbb{Z}$ acts on $A=\mathbb{R} \times \mathbb{T}^{n-1} \backslash\left\{p_{k}, k \in \mathbb{Z}\right\}$ by translation along the $\mathbb{R}$ factor, the induced action on the torus factor is trivial, and hence for all $x \in H_{i}\left(\mathbb{R} \times \mathbb{T}^{n-1}\right) \otimes \Lambda$, we have

$$
(1-t) x=0 .
$$

Since $(1-t)$ is invertible in $\Lambda$, we conclude that $x=0$, and finally, observing that $H_{i}(X)^{\mathbb{Z}}=H_{i}(X) \otimes \mathbb{Z}\left[t, t^{-1}\right]$, we have :

$$
H N_{i}(\tilde{M})=\left(H_{i}(X) \otimes \mathbb{Z}\left[t, t^{-1}\right]\right) \underset{\mathbb{Z}\left[t, t^{-1}\right]}{\otimes} \Lambda=H_{i}(X) \underset{\mathbb{Z}}{\otimes} \Lambda
$$


The universal cover $\widetilde{M}$ of $M$ can be described as a tree, with two kinds $a$ and $b$ of vertices :

- $a$-vertices are associated to copies of $\mathbb{R}^{n} \backslash \mathbb{Z}^{n}$ where the lattice corresponds to the lifts of the attaching point on the torus, and have one edge for each such point to a vertex of type $b$,

- $b$-vertices are associated to copies of $\widetilde{X} \backslash\left\{g \cdot p, g \in \pi_{1}(X)\right\}$, where $\widetilde{X}$ is the universal cover of $X$, and the points removed are the lifts of the attaching point on $X$, and have one edge for each such lift to a vertex of type $a$.

The edges then correspond to the lifts of the annulus introduced in the connected sum.

The Novikov homology $H N_{*}\left(M, u ; \mathbb{Z}\left[\pi_{1}(M)\right]\right)$ on the universal cover is less pleasant to describe than on the minimal integration cover, but we will only use the following basic facts :

Proposition 5.2. The minimal number of generators of the first Novikov homology group $H N_{1}\left(M, u ; \mathbb{Z}\left[\pi_{1}(M)\right]\right)$ associated to the universal cover is at most $\mu\left(\pi_{1}(X)\right)$, where $\mu\left(\pi_{1}(X)\right)$ is the minimal number of generators of $\pi_{1}(X)$.

If $\pi_{2}(X)=0$, then the second Novikov homology group associated to the universal cover vanishes :

$$
H N_{2}\left(M, u ; \mathbb{Z}\left[\pi_{1}(M)\right]\right)=0
$$

Remark 16. Notice that $\pi_{2}(X) \simeq H_{2}(\tilde{X} ; \mathbb{Z})$ where $\tilde{X}$ is the universal cover of $X$, and is naturally a $\mathbb{Z}\left[\pi_{1}(X)\right]$-module.

Proof. Given a family $\left(g_{1}, \ldots, g_{\mu}\right)$ that generates $\pi_{1}(X)$, for each $i$, consider a preferred lift $\gamma_{g_{i}}$ to $\widetilde{X}$ of a loop in $X$ based at the attaching point $p$ representing $g_{i}$. Similarly, in $\mathbb{R}^{n}$ consider for $1 \leq j \leq n$, the path $\gamma_{x_{j}}$, that describes the $[0,1]$-segment along the $j^{\text {th }}$ coordinate axis.

Finally, choose a preferred path $\delta:[0,+\infty) \rightarrow \mathbb{R}^{n}$ starting at the origin, such that $\lim _{t \rightarrow+\infty} x_{1}(\delta(t))=-\infty$, and that avoids $\mathbb{Z}^{n}$ for $t>0$, and use the suitable shifts of $\delta^{-1}$ and $\delta$ to extend each $\gamma_{g_{i}}$ and $\gamma_{x_{j}}$ into a path $\mathbb{R} \rightarrow \widetilde{M}$ along which the coordinate $x_{1}$ runs from and to $-\infty$ : each such path then defines a Novikov 1-cycle denoted by $\bar{\gamma}_{g_{i}}$ or $\bar{\gamma}_{x_{j}}$. Notice that $\bar{\gamma}_{x_{j}}$ and $t \cdot \bar{\gamma}_{x_{j}}$ are cobordant so that the Novikov homology class $\left[\bar{\gamma}_{x_{j}}\right]$ vanishes in $H N_{1}\left(M, u ; \mathbb{Z}\left[\pi_{1}(M)\right]\right)$.

It is not hard to see now that every Novikov 1-cycle $\sigma$ in $H N_{1}\left(M, u ; \mathbb{Z}\left[\pi_{1}(M)\right]\right)$, is cobordant to a cycle $\sigma^{\prime}$ of the form

$$
\sigma^{\prime}=\sum_{\substack{g \in \pi_{1}(M) \\ \gamma \in\left\{\gamma_{g_{i}}\right\} \cup\left\{\gamma_{x_{j}}\right\}}} n_{g} g \cdot \gamma
$$


Moreover, $\sigma^{\prime}$ being a cycle, the boundary extensions when replacing $\gamma$ by $\bar{\gamma}$ in the previous sum do not contribute, and we have

$$
\sigma^{\prime}=\sum_{\substack{g \in \pi_{1}(M) \\ \gamma \in\left\{\gamma_{g_{i}}\right\} \cup\left\{\gamma_{x_{j}}\right\}}} n_{g} g \cdot \bar{\gamma}=\sum_{\gamma \in\left\{\gamma_{g_{i}}\right\} \cup\left\{\gamma_{x_{j}}\right\}} \lambda_{\gamma} \bar{\gamma},
$$

where the $\lambda_{\gamma}$ are Novikov coefficients in $\tilde{\Lambda}$. Finally, since $\left[\bar{\gamma}_{x_{j}}\right]=0$ for each $j$, we obtain the desired result for the first Novikov homology group.

To describe the second Novikov homology group, it is convenient to use the cellular homology associated to a cell decomposition that is well suited to the connected sum. In particular, we choose a cell decomposition of $M$ in which no 2-cell meets the annulus $C=\mathbb{S}^{n-1} \times[0,1]$ introduced when performing the connected sum.

As a consequence, the 2-cells split in two disjoint families, according to the component $A=\mathbb{T}^{n} \backslash C$ or $B=X \backslash C$ of $M \backslash C$ they are contained in.

The collection of all the lifts of the cells of $M$ then defines a cell decomposition of the universal cover $\widetilde{M}$. Since the complement in $\widetilde{M}$ of the lifts of the annulus $C$ is the disjoint union $\sqcup_{\lambda} A_{\lambda} \sqcup_{\mu} B_{\mu}$ of copies of open sets $A$ and $B$ where

- $A=\mathbb{R}^{n} \backslash \mathbb{Z}^{n}$ is the universal cover $\mathbb{R}^{n}$ of $\mathbb{T}^{n}$ with the lifts of the attaching point removed.

- $B=\widetilde{X} \backslash\left\{\pi_{1}(X) \cdot p\right\}$ is the universal cover $\widetilde{X}$ of $X$ with the lifts of the attaching point removed,

we can again split the collection of 2-cells according to this collection of components.

Consider now a Novikov 2-cycle $s$ (using the cellular homology). It can be split according to the components its cells belong to :

$$
s=\sum_{\lambda} s_{\lambda}+\sum_{\mu} s_{\mu}
$$

where $s_{\lambda}$ is supported in $A_{\lambda}$ and $s_{\mu}$ in $B_{\mu}$. Then because the $A_{\lambda}$ 's and $B_{\mu}$ 's are disjoint we have :

$$
\forall \lambda, \partial s_{\lambda}=0 \quad \text { and } \quad \forall \mu, \partial s_{\mu}=0 .
$$

Moreover, a primitive $f_{\alpha}$ of a 1 -form $\alpha$ in the class $u$ is bounded on $B_{\mu}$. This implies that $s_{\mu}$ is finite, and hence defines a 2-cycle in $\tilde{X}$.

Since $H_{2}\left(B_{\mu}\right)=H_{2}(\widetilde{X})=\pi_{2}(X)=0$, this implies that $s_{\mu}$ is a boundary : there is a chain $\omega_{\mu}$ in $B_{\mu}$ such that $s_{\mu}=\partial \omega_{\mu}$.

The chain $\omega=\sum_{\mu} \omega_{\mu}$ is then a Novikov chain (above any level, there are only finitely many $\mu$ such that $s_{\mu} \neq 0$, so that $\omega$ is finite above any level), and

$$
\partial \omega=\sum_{\mu} s_{\mu}
$$


In particular, the homology class $[s]$ can be written as

$$
[s]=\sum_{\lambda}\left[s_{\lambda}\right]
$$

On the other hand, denote by $t_{1} \in \pi_{1}(M)$ the loop associated to the first coordinate in the torus. It acts on each $A_{\lambda}$ as the translation along $(1,0, \ldots, 0)$, and any Novikov 2-cycle $\tau$ in $A_{\lambda}$ is homologous to $t_{1} \cdot \tau$ (this is clear in $\mathbb{R}^{n}$, and the points that are removed from $A_{\lambda}$ are sufficiently high codimensional to be avoided).

As a consequence, for all $\lambda$ :

$$
t_{1} \cdot\left[s_{\lambda}\right]=\left[s_{\lambda}\right],
$$

(where the brackets denote the homology class). We conclude that

$$
\left(1-t_{1}\right) \cdot\left[s_{\lambda}\right]=0
$$

and since $\left(1-t_{1}\right)$ is invertible in the Novikov ring, $\left[s_{\lambda}\right]=0$, and finally $[s]=0$.

5.2. Novikov fundamental group for $\mathbb{T}^{n} \sharp X$. In this section we compute and investigate the Novikov fundamental group associated to the minimal integration cover in the particular situation described above where $M=$ $\mathbb{T}^{n} \sharp X$. For convenience, the fundamental group of $X$ will be denoted by $G$.

5.2.1. Computation of the Novikov fundamental group. The Novikov fundamental group $\pi_{1}\left(\mathbb{T}^{n} \sharp X, u\right)$ naturally appears as a completion of the free product of infinitely many copies of $G$. More precisely, consider the infinite sequence of groups $\left(G_{k}\right)_{k \in \mathbb{Z}}$ where all the groups $G_{k}$ are copies of $G$, and define for each $h \in \mathbb{Z}$ the group

$$
\Pi_{h}=\underset{k \geq h}{*} G_{k} .
$$

For two integers $h, h^{\prime}$ with $h<h^{\prime}$, we have

$$
\Pi_{h^{\prime}}=\Pi_{h} /\left(G_{k}=1, h \leq k<h^{\prime}\right) .
$$

Hence there are projections

$$
\zeta_{h}^{h^{\prime}}: \Pi_{h} \rightarrow \Pi_{h^{\prime}}
$$

such that for $h<h^{\prime}<h^{\prime \prime}$, we have :

$$
\zeta_{h}^{h^{\prime \prime}}=\zeta_{h^{\prime}}^{h^{\prime \prime}} \circ \zeta_{h}^{h^{\prime}}
$$

Define then

$$
\Pi=\lim _{h} \Pi_{h}
$$

Proposition 5.3. With the above notations, $\pi_{1}(\tilde{M}, u)=\Pi$. 
Proof. Pick a 1-form $\alpha$ that "separates" the different copies of $X$, i.e. such that a primitive $f_{\alpha}$ has a level $h_{0}$, that can be supposed to be 0 , that does not touch any lift of $X$ nor of the annulus where the connected sum takes place.

By rescaling the cohomology class $u$, we can suppose it has period 1, i.e. that $\alpha\left(\pi_{1}(M)\right)=\mathbb{Z}$, so that the integral levels of $f_{\alpha}$ separate the different lifts of $X$.

Then for any $h \in \mathbb{Z}$, we have

$$
\left\lfloor\pi_{1}\left(f_{\alpha}\right)\right\rfloor_{h}=\pi_{1}\left(\lfloor\tilde{M}\rfloor_{h}\right)=\underset{k \geq h}{*} \pi_{1}(X)=\Pi_{h} .
$$

Since the projections $\lfloor\tilde{M}\rfloor_{h} \rightarrow\lfloor\tilde{M}\rfloor_{h^{\prime}}$ given by inclusion of sublevels induce the same maps $\zeta_{h}^{h^{\prime}}$ as in (5.2) , we derive $\pi_{1}(\tilde{M}, u)=\Pi$.

With the notations above we obviously have

$$
\mu_{D T C}\left(\pi_{1}(\tilde{M}, u)\right) \leq \mu\left(\pi_{1}(X)\right),
$$

where $\mu\left(\pi_{1}(X)\right)$ is the minimal number of generators of $\pi_{1}(X)$.

This estimate is straightforward, but the reverse inequality, which we expect to hold, is surprisingly far from obvious. We will limit ourselves to the following statement :

Proposition 5.4. Unless $\pi_{1}(X)$ is a cyclic group, $\mu_{D T C}\left(\pi_{1}(\tilde{M}, u)\right) \geq 2$. In particular, when $X=S \times \mathbb{S}^{n-3}$, we have $\mu_{D T C}\left(\pi_{1}(\tilde{M}, u)\right)=2$.

Remark 17. From the usual group theory point of view, deck transformations turn a single generator "up to DTC" into an infinite collection of generators. Therefore, telling if a group has more than one generator up to DTC is far from obvious in general.

To emphasize this issue, consider the same question for the infinite cartesian product $\Pi^{\prime}=G^{\mathbb{Z}}$ instead of the free product : when $G$ is the group given in (5.1) for instance, $\Pi^{\prime}$ has a single generator up to shift while neither $G$ nor $\Pi^{\prime}$ are cyclic...

The end of this section is dedicated to the proof of proposition 5.4.

Definition 5.5. Given a collection of groups $G_{1}, \ldots, G_{N}$, we say that a reduced sequence is a sequence $g_{1}, \ldots, g_{m}$ such that for $1 \leq i \leq m$ we have $g_{i} \in G_{k}$ for some $k, g_{i} \neq 1$ and $g_{i}, g_{i+1}$ are not in the same factor $G_{l}$ for some l.

We say that $g=g_{1} \ldots g_{m} \in *_{1 \leq k \leq N} G_{k}$ is in the normal form if $g_{1}, \ldots, g_{m}$ is a reduced sequence. According to the Normal Form Theorem for free product of groups, every element of the free product $*_{1 \leq k \leq N} G_{k}$ can be written in a unique way in a normal form.

Definition 5.6. An element $g$ in a free product of groups $*_{1 \leq k \leq N} G_{k}$ is said to be a single letter (resp. non trivial single letter) if its normal form has length $\leq 1$ (resp. 1), i.e. if $g \in G_{k_{0}} \subset *_{1 \leq k \leq N} G_{k}$ (resp. $g \in G_{k_{0}} \backslash\{1\}$ ) for some index $k_{0}$. 
Similarly, an element $g$ in $\Pi$ will be said to be a single letter if $g \in G_{k} \subset \Pi$ for some $k$.

Lemma 5.7. Let $g \in *_{k=1}^{N} G_{k}$ be an element of a finite free product of groups. If $g^{\nu}$ is a single letter for some $\nu \neq 0$, then $g$ itself is a single letter.

Proof. Let $g=g_{1} \ldots g_{N}$ be in the normal form.

Let $k$ be the maximal index such that $\left(g_{1} \ldots g_{k}\right)=\left(g_{N-k+1} \ldots g_{N}\right)^{-1}$. Then letting $w=g_{1} \ldots g_{k}$ and $g^{\prime}=g_{k+1} \ldots g_{N-k}$ (notice that $g^{\prime} \neq 1$ ), we have $g=w g^{\prime} w^{-1}$ and the normal form of $g^{\nu}$ is

$$
g^{\nu}=w g^{\prime \nu} w^{-1} \text {. }
$$

For $g^{\nu}$ to be a single letter hence requires that $w=1$ and $g^{\prime}$ to be a single letter, which means that $g$ itself is a single letter.

Remark 18. Notice for future use that the proof also shows that if $g$ is not a single letter, the first and last letters of the normal form of any non trivial power of $g$ are the same as that of $g$ or $g^{-1}$.

Lemma 5.8. Suppose $g \in \Pi=*_{k \in \mathbb{Z}} G_{k}$ is such that the sub-group generated by $g$ up to DTC contains a non trivial single letter $a \in G_{k_{0}} \backslash\{1\}$. Let $h_{0}$ be the first level above which $\zeta_{\infty}^{h_{0}}(g) \neq 1$. Then the normal form of $\zeta_{\infty}^{h_{0}}(g)$ is a single letter.

Proof. By assumption, there are shifts of powers of $g$ such that above the level $k_{0}$ of this single letter $a$, we have :

$$
a=\zeta_{\infty}^{k_{0}}\left(\prod_{k=1}^{N} s^{d_{k}}\left(g^{\nu_{k}}\right)\right)
$$

where $s$ denotes the upward shift. Since everything is defined up to shift, we can suppose that $d=\max \left\{d_{k}, 1 \leq k \leq N\right\}=0$. Then $k_{0} \leq h_{0}$, so that

$$
\zeta_{\infty}^{h_{0}}\left(\prod_{k=1}^{N} s^{d_{k}}\left(g^{\nu_{k}}\right)\right)=\zeta_{\infty}^{h_{0}}(a)= \begin{cases}a & \text { if } k_{0}=h_{0}, \\ 1 & \text { if } k_{0}<h_{0} .\end{cases}
$$

In both cases, $\zeta_{\infty}^{h_{0}}(a)$ is a single, possibly trivial, letter $b$.

But for all $d<0$, we have $\zeta_{\infty}^{h_{0}}\left(s^{d} g\right)=1$, so that (5.3) reduces to

$$
\zeta_{\infty}^{h_{0}}\left(g^{\nu}\right)=b=\left[\zeta_{\infty}^{h_{0}}(g)\right]^{\nu},
$$

where $\nu=\sum_{k / d_{k}=0} \nu_{k}$. From lemma 5.7 it follows that $\zeta_{\infty}^{h_{0}}(g)$ is a single letter, which is non trivial by the definition of $h_{0}$.

Proof of proposition 5.4. Suppose we can find a single generator $g$ for $\Pi$ up to DTC.

Let $h_{0}$ be the first level above which $\zeta_{\infty}^{h_{0}}(g) \neq 1$. From lemma 5.8 it follows that

$$
\zeta_{\infty}^{h_{0}}(g)=s^{h_{0}}(a)
$$

is a single letter $a$ in level $h_{0}$. Here $G$ is identified with $G_{0}$. 
Let then $h_{1}$ be the first level above which the normal form of $g$ contains a letter that is not a power of $a$ :

$$
\begin{gathered}
\forall k \in\left\{h_{1}+1, \ldots, h_{0}\right\}, \zeta_{\infty}^{k}(g) \in \underset{h_{1}<h}{*}\left(s^{h}(a)\right)^{\mathbb{Z}}, \\
\zeta_{\infty}^{h_{1}}(g) \notin \underset{h_{1} \leq h}{*}\left(s^{h}(a)\right)^{\mathbb{Z}} .
\end{gathered}
$$

Let then

$$
\omega=\zeta_{\infty}^{h_{1}}(g) \in \underset{k \geq h_{1}}{*} G_{k}
$$

Observe that $\omega$ is not a single letter, and hence none of its non trivial powers either. The normal form of $\omega$

$$
\omega=s^{k_{0}}\left(b_{0}\right) s^{k_{1}}\left(b_{1}\right) \ldots s^{k_{N}}\left(b_{N}\right)
$$

is a sequence of letters $b_{i} \in G \backslash\{1\}$ in levels $k_{i}$ such that $k_{i} \geq h_{1}$ and $|i-j|=1 \Rightarrow k_{i} \neq k_{j}$.

Let $A_{-}$and $A_{+}$be the longest possible words at the beginning and at the end of $\omega$ whose letters are all powers of $a$ in a level $<h_{0}$. More precisely, let :

$$
A_{-}=\prod_{0 \leq i<i_{-}} s^{k_{i}}\left(b_{i}\right), \quad \omega^{\prime}=\prod_{i_{-} \leq i \leq i_{+}} s^{k_{i}}\left(b_{i}\right), \quad A_{+}=\prod_{i_{+}<i \leq N} s^{k_{i}}\left(b_{i}\right),
$$

where $i_{-}=\inf \left\{i, b_{i} \notin a^{\mathbb{Z}}\right.$ or $\left.k_{i}=h_{0}\right\}$ and $i_{+}=\sup \left\{i, b_{i} \notin a^{\mathbb{Z}}\right.$ or $\left.k_{i}=h_{0}\right\}$. Then

$$
\omega=A_{-} \omega^{\prime} A_{+}
$$

and the first and last letters of $\omega^{\prime}, s^{k_{i_{-}}}\left(b_{i_{-}}\right)$and $s^{k_{i_{+}}}\left(b_{i_{+}}\right)$and are such that:

$$
\begin{aligned}
& k_{i_{-}} \geq h_{0} \text { or } \forall \alpha \in \mathbb{Z}, a^{\alpha} \neq 1 \Rightarrow a^{\alpha} b_{i_{-}} \neq 1, \\
& k_{i_{+}} \geq h_{0} \text { or } \forall \alpha \in \mathbb{Z}, a^{\alpha} \neq 1 \Rightarrow b_{i_{+}} a^{\alpha} \neq 1 .
\end{aligned}
$$

Moreover, if $k_{i_{-}}=k_{i_{+}}$and $\exists \alpha \in \mathbb{Z}, b_{i_{+}} a^{\alpha} b_{i_{-}}=1$, we replace $b_{i_{-}}$by $a^{\alpha} b_{i_{-}}$ and $A_{-}$by $A_{-} s^{k_{i_{-}}}\left(a^{-\alpha}\right)$, so that $b_{i_{+}} b_{i_{-}}=1$, and hence

$$
k_{i_{-}} \neq k_{i_{+}} \text {or } \forall \alpha \in \mathbb{Z}, a^{\alpha} \neq 1 \Rightarrow b_{i_{+}} a^{\alpha} b_{i_{-}} \neq 1 .
$$

Finally, recall for future use that from remark 18, the first and last letters $b_{i_{-}}$and $b_{i_{+}}$of $\omega^{\prime}$ are also the first and last letters of any non trivial power of $\omega^{\prime}$.

Since $G$ is not cyclic, there is an element

$$
c \in G \backslash\left\{a^{\mathbb{Z}}\right\}
$$

which we regard as a single letter in $G_{0} \subset \Pi$. Since $g$ generates $\Pi$ up to DTC, there is a word $x=\prod_{i} s^{d_{i}}\left(g^{\nu_{i}}\right)$ such that

$$
\zeta_{\infty}^{0}(x)=c \in G_{0} .
$$

Observe that $d=\max \left(d_{i}\right)$ satisfies $d+h_{1} \geq 0$, since otherwise all the letters in $\zeta_{\infty}^{0}(x)$ would belong to $a^{\mathbb{Z}}$, which contradicts (5.8). To reduce notations, 
and since everything is defined up to shift, we shift everything by $-d$, so that we can consider that $d=0$ and $c$ belongs to the level $-d$, which is not higher than $h_{1}$.

Then the normal form of $\zeta_{\infty}^{h_{1}}(x)$ is either 1 (if $-d<h_{1}$ ) or the single letter $c\left(\right.$ if $\left.-d=h_{1}\right)$ :

$$
\zeta_{\infty}^{h_{1}}(x)=1 \quad \text { or } \quad \zeta_{\infty}^{h_{1}}(x)=c \in G_{h_{1}}
$$

On the other hand, $\zeta_{\infty}^{h_{1}}(x)$ expands as

$$
\zeta_{\infty}^{h_{1}}(x)=\prod_{i} \zeta_{\infty}^{h_{1}}\left(s^{d_{i}}(g)\right)^{\nu_{i}}
$$

and when $d_{i}<0$, the normal form of $\zeta_{\infty}^{h_{1}}\left(s^{d_{i}}(g)\right)$ is a word whose letters are all powers of $a$ in levels in $\left\{h_{1}, \ldots, h_{0}-1\right\}$, while for $d_{i}=0$, we have $\zeta_{\infty}^{h_{1}}\left(s^{d_{i}}(g)\right)=\omega$. As a consequence, we can write $\zeta_{\infty}^{h_{1}}(x)$ as

$$
\zeta_{\infty}^{h_{1}}(x)=A_{0} \omega^{\beta_{1}} A_{1} \ldots \omega^{\beta_{N^{\prime}}} A_{N^{\prime}},
$$

where $\omega^{\beta_{i}} \neq 1$ for $1 \leq i \leq N^{\prime}$ and $N^{\prime}$ is indeed at least 1 because of assumption (5.8).

$$
A_{i} \in \underset{h_{1} \leq k<h_{0}}{*}\left(s^{k}(a)\right)^{\mathbb{Z}} .
$$

Using (5.4) and merging the $A_{i}$ and the possible $A_{-}$or $A_{+}$, we can rewrite this as

$$
\zeta_{\infty}^{h_{1}}(x)=A_{0}^{\prime} \omega^{\prime \beta_{1}^{\prime}} A_{1}^{\prime} \ldots \omega^{\prime \beta_{N^{\prime \prime}}^{\prime}} A_{N^{\prime \prime}}^{\prime},
$$

where the $A_{i}^{\prime}$ also satisfy (5.9) and

$$
A_{i}^{\prime} \neq 1 \text { for } 0<i<N^{\prime \prime} \text {. }
$$

Observe now that from (5.5), (5.6), and (5.7), the normal form of $\zeta_{\infty}^{h_{1}}(x)$ is obtained from the concatenation of the normal form of all the $A_{i}^{\prime}$ and $\omega^{\prime \beta_{j}^{\prime}}$ given by (5.10) only by merging the first and/or last letter of $A_{i}^{\prime}$ with the last and/or first letter of its neighbors if ever possible.

In particular, the collection of levels supporting non trivial letters in the normal form of $\zeta_{\infty}^{h_{1}}(x)$ is the union of the levels supporting non trivial letters in the normal form of all the $A_{i}^{\prime}$ and $\omega^{\prime \beta_{i}^{\prime}}$.

Since none of the $\omega^{\prime \beta_{i}^{\prime}}$ are single letters, this collection contains at least two levels and $\zeta_{\infty}^{h_{1}}(x)$ cannot be a single letter, which is a contradiction.

5.2.2. An upper bound for the deficiency. Estimating $\mu_{D T C}$ and $\rho_{D T C}$ is hard in general, but the object of this section is to give an upper bound for the deficiency of $\Pi$ up to DTC, i.e. of the maximum of the difference $m-r$ of the number of generators $m$ and relations $r$ in a finite presentation up to DTC.

For the group $\pi_{1}(X)$ itself, the deficiency is bounded above by the dimension of the real vector space

$$
L=\operatorname{Hom}\left(\pi_{1}(X), \mathbb{R}\right)
$$


of group morphisms from $\pi_{1}(X)$ to $(\mathbb{R},+)$. The naive Novikov counterpart of this space would be the space

$$
L_{\Lambda_{\mathbb{R}}}=\operatorname{Hom}^{\mathcal{D}}\left(\pi_{1}(\tilde{M}, u), \Lambda_{\mathbb{R}}\right)
$$

of shift equivariant morphisms from $\pi_{1}(\tilde{M}, u)$ to the field

$$
\Lambda_{\mathbb{R}}=\mathbb{R}((t))
$$

of Laurent formal series, seen as an additive group. However, we need to keep track of the filtration and we restrict attention to morphisms that are compatible with the inverse systems in the following way.

First observe that $\Lambda_{\mathbb{R}}$ is in fact the inverse limit of the inverse system

$$
\cdots \stackrel{[\cdot]_{d+1}}{\longrightarrow} \mathbb{R}_{d+1}((t)) \stackrel{[\cdot]_{d}}{\longrightarrow} \mathbb{R}_{d}((t)) \stackrel{[\cdot]_{d-1}}{\longrightarrow} \cdots
$$

where $\mathbb{R}_{d}((t))$ denotes the Laurent polynomials of degree at most $d$, and the transition maps are given by the truncation of high degree terms. For convenience, we will write $\Lambda_{d}=\mathbb{R}_{d}((t))$. Then :

$$
\Lambda_{\mathbb{R}}=\lim _{d \rightarrow+\infty} \Lambda_{d}
$$

Let then

$$
L_{\Lambda_{\mathbb{R}}}=\underline{\operatorname{Hom}}^{\mathcal{D}}(\Pi, \Lambda)
$$

be the space of shift-equivariant morphisms from $\Pi$ to $\Lambda_{\mathbb{R}}$ that are limits of morphisms from the inverse system $\left(\Pi_{h}\right)_{h \in \mathbb{Z}}$ to the inverse system $\left(\Lambda_{d}\right)_{d \in \mathbb{Z}}$, i.e. the space of morphisms $\phi: \Pi \rightarrow \Lambda_{\mathbb{R}}$ such that there is a constant $d_{0} \in \mathbb{Z}$ and a collection $\left(\phi_{h}\right)_{h \in \mathbb{Z}}$ with $\phi_{h} \in \operatorname{Hom}\left(\Pi_{h}, \Lambda_{d_{0}-h}\right)$, such that

(1) the following diagram

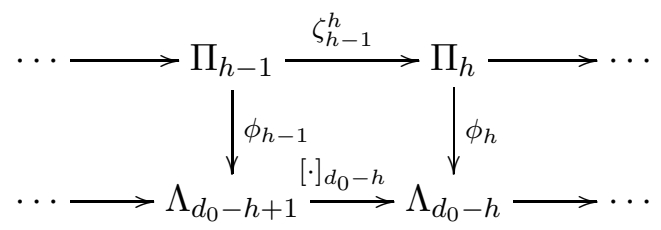

is commutative,

(2) each $\phi_{h}$ is shift equivariant:

$$
\forall g \in \Pi_{h}, \phi_{h}(s(g))=t^{-1} \phi_{h-1}(g),
$$

(3) and

$$
\phi=\lim _{h} \phi_{h}
$$

Here, $\Pi_{h}=*_{k \geq h} G_{k}$ and $s$ denotes the map $\Pi_{h-1} \rightarrow \Pi_{h}$ induced by the positive shift.

Given an element $\phi$ which is the limit of a morphism

$$
\left(\phi_{h}: \Pi_{h} \rightarrow \Lambda_{d_{0}-h}\right)_{h \in \mathbb{Z}} \in L_{\Lambda_{\mathbb{R}}},
$$


and $\lambda \in \Lambda_{\mathbb{R}}$, the morphisms

$$
\begin{aligned}
\psi_{h}: \Pi_{h} & \rightarrow \Lambda_{d_{0}-\nu-h} \\
g & \mapsto\left[\lambda \phi_{h}(g)\right]_{d_{0}-\nu-h}
\end{aligned}
$$

where $\nu$ is the $t$ valuation of $\lambda$, define a new element $\psi=\lambda \phi$ in $L_{\Lambda_{\mathbb{R}}}$ that only depends on $\phi$ and not on the choice of $d_{0}$ and $\left(\phi_{h}\right)_{h}$. Endowed with this operation, it is not hard to check that :

Proposition 5.9. The space $L_{\Lambda_{\mathbb{R}}}$ is a $\Lambda_{\mathbb{R}}$-vector space.

Recall that a finite presentation $<g_{1}, \ldots, g_{m} \mid w_{1}, \ldots, w_{r}>$ of $G=\pi_{1}(X)$, allows to identify $L$ as the kernel of a linear map

$$
0 \rightarrow L \rightarrow \mathbb{R}^{m} \rightarrow \mathbb{R}^{r} \rightarrow 0
$$

defined by the abelian rewriting of the words $w_{1}, \ldots, w_{r}$.

To establish a similar statement for $L_{\Lambda_{\mathbb{R}}}$, some restriction on the family of generators is required. Observe that the kernels $K_{h}$ of the evaluation maps $\mathbb{F}_{h} \stackrel{\mathrm{ev}_{h}}{\longrightarrow} \Pi_{h}$ defined in (2.5) form an inverse system.

Definition 5.10. A presentation up to DTC of $\pi_{1}(\tilde{M}, u)$ is said to have well behaved relations if the inverse system $\left(K_{h}\right)$ satisfies the Mittag-Leffler condition.

This condition is not restrictive for the Morse interpretation of the Novikov fundamental group since it always gives well-behaved relations :

Proposition 5.11. The presentation of $\pi_{1}(\tilde{M}, u)$ up to DTC associated to the index 1 and 2 critical points of a Morse 1-form $\alpha$ in the class $u$ always has well behaved relations.

Proof. The proof of statement 3.5 shows that the maps $K_{h} \rightarrow K_{h^{\prime}}$ are in fact surjective.

We can now generalize (5.11) to the Novikov situation.

Lemma 5.12. Let $\left(g_{1}, \ldots, g_{m}\right)$ and $\left(w_{1}, \ldots, w_{r}\right)$ be a presentation up to DTC of $\Pi=\pi_{1}(\tilde{M}, u)$ with well-behaved relations. Then there is a linear map $\Lambda_{\mathbb{R}}^{m} \rightarrow \Lambda_{\mathbb{R}}^{r}$ whose kernel is $L_{\Lambda_{\mathbb{R}}}$, i.e. there is a short exact sequence

$$
0 \rightarrow L_{\Lambda_{\mathbb{R}}} \stackrel{\epsilon}{\rightarrow} \Lambda_{\mathbb{R}}^{m} \stackrel{\rho}{\rightarrow} \Lambda_{\mathbb{R}}^{r}
$$

In particular, $\operatorname{dim}_{\Lambda_{\mathbb{R}}} L_{\Lambda_{\mathbb{R}}} \geq m-r$.

Proof. The map $\epsilon$ is defined by evaluating a morphism $\phi \in L_{\Lambda_{\mathbb{R}}}$ on $\left(g_{1}, \ldots, g_{m}\right)$ :

$$
\epsilon(\phi)=\left(\phi\left(g_{1}\right), \ldots, \phi\left(g_{m}\right)\right) .
$$

To check it is injective, consider some $\phi \in \operatorname{ker} \epsilon$, and a sequence

$$
\left(\phi_{h}: \Pi_{h} \rightarrow \Lambda_{d_{0}-h}\right)_{h \in \mathbb{Z}}
$$

defining it. Since $\phi$ is supposed to be shift equivariant, we have then $\phi_{h}\left(\zeta_{\infty}^{h}\left(s^{k}\left(g_{i}\right)\right)\right)=0$ for all $i \in\{1, \ldots, m\}$ and all $k \in \mathbb{Z}$. Since $\left(g_{1}, \ldots, g_{m}\right)$ 
generates $\Pi$ up to DTC, this means that $\phi_{h}$ vanishes on the image of $\Pi \stackrel{\zeta_{\infty}^{h}}{\longrightarrow} \Pi_{h}$ and hence that $\phi=0$.

The definition of the map $\rho$ requires some extra care because of the completion process involved in the definition of $\overline{F_{\mathbb{Z} \times\left\{g_{1}, \ldots, g_{m}\right\}}}$. Recall that the deck transformation group $\mathcal{D}$ is isomorphic to $\mathbb{Z}$ in our case. The completion process rests on a notion of height for the letters, and in order to keep the notations reasonably simple, following remark 9, we fix the height of all the letters $g_{1}, \ldots, g_{m}$ to be 0 and assume that the downward shift decreases the height by 1 . The associated restriction maps, in the completion of the free group, will still be denoted by $\zeta_{\infty}^{h}$ :

$$
\overline{F_{\mathbb{Z} \times\left\{g_{1}, \ldots, g_{m}\right\}}} \stackrel{\zeta_{\infty}^{h}}{\longrightarrow}\left\lfloor F_{\mathbb{Z} \times\left\{g_{1}, \ldots, g_{m}\right\}}\right\rfloor_{h} .
$$

Let $w$ be one of the relations $w_{i}, 1 \leq i \leq r$. It is an element of the projective limit $\lim _{\longleftarrow}\left\lfloor F_{\mathbb{Z} \times\left\{g_{1}, \ldots, g_{m}\right\}}\right\rfloor_{h}$, and hence, above each level $h, \zeta_{\infty}^{h} w$ is a finite word in shifts of the letters $g_{1}, \ldots, g_{m}$. Using the notations of section 2.5.2, we can write

$$
\zeta_{\infty}^{h}(w)=\prod_{j=1}^{N}\left(k_{j}, g_{i_{j}}^{\alpha_{j}}\right),
$$

where the shift components $k_{j}$ are such that $k_{j} \geq h$.

Given an element $\lambda=\left(\lambda_{1}, \ldots, \lambda_{m}\right) \in \Lambda_{\mathbb{R}}^{m}$, we define an element $\rho_{w}(\lambda) \in$ $\Lambda_{\mathbb{R}}$ in the following way.

If $\lambda=0$, we simply let $\rho_{w}(\Lambda)=0$. If not, consider an integer $\nu$ such that

$$
\nu \leq \min \left\{\nu\left(\lambda_{i}\right): \lambda_{i} \neq 0\right\},
$$

where $\nu\left(\lambda_{i}\right)$ denotes the $t$-valuation of $\lambda_{i}$. For $h \in \mathbb{Z}$, define

$$
\rho_{w, h}(\lambda)=\left[\sum_{j=1}^{N} \alpha_{j} t^{-k_{j}} \lambda_{i_{j}}\right]_{\nu-h} \in \Lambda_{\nu-h} .
$$

Recall that for $h<h^{\prime}$, we have

$$
\zeta_{\infty}^{h^{\prime}}(w)=\zeta_{h}^{h^{\prime}}\left(\zeta_{\infty}^{h}(w)\right)
$$

i.e. that $\zeta_{\infty}^{h^{\prime}}(w)$ is obtained from $\zeta_{\infty}^{h}(w)$ by removing the letters whose levels are in $\left\{h, \ldots, h^{\prime}-1\right\}$. In particular, we have :

$$
\begin{aligned}
{\left[\rho_{w, h}(\lambda)\right]_{\nu-h^{\prime}} } & =\left[\sum_{\substack{1 \leq j \leq N \\
k_{j}<h^{\prime}}} \alpha_{j} t^{-k_{j}} \lambda_{i_{j}}\right]_{\nu-h^{\prime}}+\left[\sum_{\substack{1 \leq j \leq N \\
k_{j} \geq h^{\prime}}} \alpha_{j} t^{-k_{j}} \lambda_{i_{j}}\right]_{\nu-h^{\prime}} \\
& =\left[\sum_{\substack{1 \leq j \leq N \\
k_{j} \geq h^{\prime}}} \alpha_{j} t^{-k_{j}} \lambda_{i_{j}}\right]_{\nu-h^{\prime}} \\
& =\rho_{w, h^{\prime}}(\lambda),
\end{aligned}
$$


which means that the sequence $\left(\rho_{w, h}(\lambda)\right)$ defines an element $\rho_{w}(\lambda)$ in the inverse limit $\Lambda_{\mathbb{R}}$.

Observe moreover that this limit does not depend on the choice of $\nu$ provided $\nu$ satisfies (5.13). In particular, an integer $\nu$ that suits two different $\lambda$ can always be found, i.e. for $\lambda, \lambda^{\prime}$ there exists

$$
\nu \leq \min \left\{\nu\left(\lambda_{i}\right), \nu\left(\lambda_{i}^{\prime}\right): \lambda_{i}, \lambda_{i}^{\prime} \neq 0\right\},
$$

and it is not hard to check that the map

$$
\begin{array}{ccc}
\Lambda_{\mathbb{R}}^{m} & \stackrel{\rho_{w}}{\longrightarrow} \Lambda_{\mathbb{R}} \\
\left(\lambda_{1}, \ldots, \lambda_{m}\right) & \mapsto & \rho_{w}(\lambda) .
\end{array}
$$

is $\Lambda_{\mathbb{R}}$ linear.

We still have to prove the exactness of (5.12). Since each $w_{i}$ is a relation, we have $\rho \circ \epsilon=0$. Conversely, consider some $\left(\lambda_{1}, \ldots, \lambda_{m}\right) \in \operatorname{ker} \rho$. Fix some integer $\nu$ satisfying (5.13), and consider the morphisms

$$
\begin{array}{ccc}
F_{h} & \stackrel{\tilde{\phi}_{h}}{\longmapsto} & \Lambda_{\nu-h} \\
\prod_{j} s^{k_{j}}\left(g_{i_{j}}^{\alpha_{j}}\right) & \mapsto & \sum_{j} \alpha_{j} t^{-k_{j}} \lambda_{i_{j}}
\end{array} .
$$

Since $\lambda \in \operatorname{ker} \rho$, we have

$$
\mathcal{R}_{h} \subset \operatorname{ker} \tilde{\phi}_{h},
$$

where $\mathcal{R}_{h}=\zeta_{\infty}^{h} \mathcal{R}(A)$.

Recall that, since the maps $\Pi_{h} \rightarrow \Pi_{h+1}$ are surjective and $A$ generates $\Pi$ up to DTC, every element $g \in \Pi_{h}$ can be written in the form

$$
g=\prod_{j=1}^{N} \zeta_{\infty}^{h}\left(s^{k_{j}}\left(g_{i_{j}}^{\alpha_{j}}\right)\right) .
$$

In particular, this means that the projection $F_{h} \rightarrow \Pi_{h}$ is surjective.

Notice however that although $\mathcal{R}_{h} \subseteq \operatorname{ker}\left(F_{h} \stackrel{\mathrm{ev}_{h}}{\longrightarrow} \Pi_{h}\right)$, the inclusion might be strict in general, so that $\tilde{\phi}$ may not induce a morphism on $\Pi_{h}$ in general.

The assumption that the relations are well behaved is made precisely to fix this : let $\kappa$ be a constant such that

$$
\forall h^{\prime} \in \mathbb{Z}, \forall h \in \mathbb{Z}, h \leq h^{\prime}-\kappa \Rightarrow \zeta_{h}^{h^{\prime}}\left(K_{h}\right)=\zeta_{h^{\prime}-\kappa}^{h^{\prime}}\left(K_{h^{\prime}-\kappa}\right),
$$

where $K_{h}=\operatorname{ker}\left(F_{h} \stackrel{\mathrm{ev}_{h}}{\longrightarrow} \Pi_{h}\right)$. Then, for $h \leq h^{\prime}-\kappa$, we have

$$
\zeta_{h}^{h^{\prime}}\left(K_{h}\right)=\zeta_{\infty}^{h^{\prime}}(\mathcal{R}(A))=\mathcal{R}_{h^{\prime}}
$$

Observe now that $\zeta_{h-\kappa}^{h}: \Pi_{h-\kappa} \rightarrow \Pi_{h}$ has a natural section

$$
\Pi_{h-\kappa} \stackrel{\iota}{\underset{\zeta_{h-\kappa}^{h}}{\longrightarrow}} \Pi_{h}
$$


mapping $\Pi_{h}$ to $\{1\} * \cdots *\{1\} * \Pi_{h} \subset \Pi_{h-\kappa}$. Let $F_{h-\kappa}^{\prime}=\mathrm{ev}_{h-\kappa}^{-1}\left(\iota\left(\Pi_{h}\right)\right.$ and $F_{h}^{\prime \prime}=\zeta_{h-\kappa}^{h}\left(F_{h-\kappa}^{\prime}\right):$

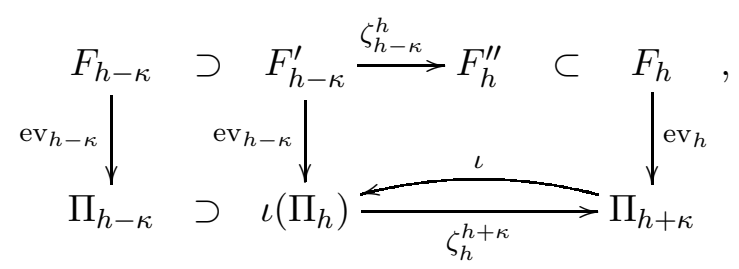

and consider the restriction $\mathrm{ev}_{h}^{\prime}$ of $\mathrm{ev}_{h}$ to $F_{h}^{\prime}$. By construction, $\mathrm{ev}_{h}^{\prime}$ is surjective, and its kernel satisfies :

$$
\operatorname{ker}\left(\operatorname{ev}_{h}^{\prime}\right)=\zeta_{h-\kappa}^{h}\left(F_{h-\kappa}^{\prime} \cap K_{h-\kappa}\right) \subset \mathcal{R}_{h} .
$$

As a consequence, the morphism $\tilde{\phi}_{h}: F_{h}^{\prime \prime} \rightarrow \Lambda_{\nu-h}$ satisfies

$$
\operatorname{ker}\left(\operatorname{ev}_{h}^{\prime}\right) \subset \mathcal{R}_{h} \subset \operatorname{ker}(\tilde{\phi})
$$

and hence induces a morphism $\phi_{h}: \Pi_{h}=F_{h}^{\prime \prime} / \mathrm{ker} \mathrm{ev}_{h}^{\prime} \rightarrow \Lambda_{\nu-h}$. Moreover, by construction we have

$$
\phi_{h}\left(g_{i}\right)=\zeta_{\infty}^{\nu-h}\left(\lambda_{i}\right)
$$

As a consequence, the collection of morphisms $\left(\phi_{h}\right)_{h \in \mathbb{Z}}$ defines an element $\phi \in L_{\Lambda_{\mathbb{R}}}$ such that

$$
\lambda=\epsilon(\phi)
$$

Proposition 5.13. We have $L_{\Lambda_{\mathbb{R}}}=L \otimes \Lambda_{\mathbb{R}}$. In particular,

$$
\operatorname{dim}_{\mathbb{R}} L=\operatorname{dim}_{\Lambda_{\mathbb{R}}} L_{\Lambda_{\mathbb{R}}} .
$$

Proof. Let $\phi$ be a morphism in $L_{\Lambda_{\mathbb{R}}}$. Observe that a morphism $\phi=\left(\phi_{h}\right)_{h \in \mathbb{Z}} \in$ $L_{\Lambda_{\mathbb{R}}}$ is fully determined by its behaviour on single letters in $G_{0} \subset \Pi$, i.e. by the maps

$$
\phi_{h, 0}=\phi_{\left.h\right|_{G_{0}}}: G_{0} \rightarrow \Lambda_{h} .
$$

Splitting $\phi_{h, 0}$ according to the degree of $t$, we can write

$$
\phi_{h, 0}=\sum_{d=-N}^{-h} t^{d} \alpha_{h, d},
$$

where the $\alpha_{h, d}$ are now morphisms from $G_{0}$ to $\mathbb{R}$, i.e. elements of $L$.

Splitting each $\alpha_{h, d}$ according to a basis $\left(\alpha^{(1)}, \ldots, \alpha^{(k)}\right)$ of $L$, we write $\phi_{h, 0}$ as

$$
\phi_{h, 0}=\sum_{i=1}^{k} \lambda_{i, h} \alpha^{(i)},
$$


where each $\lambda_{i, h}$ is in $\Lambda_{-h}$. Moreover, for $h<h^{\prime}, \lambda_{h^{\prime}}=\left[\lambda_{h}\right]_{-h^{\prime}}$, so that the sequence $\lambda_{i, h}$ defines an element $\lambda_{i} \in \Lambda_{\mathbb{R}}$, and we have

$$
\phi=\sum_{i=1}^{k} \lambda_{i} \alpha^{(i)} .
$$

Here $\alpha^{(i)}$ is seen as the element of $L$ naturally induced by $\alpha^{(i)}$ on the 0-level. This ends the proof of 5.13 .

Corollary 5.14. The real dimension $\operatorname{dim}_{\mathbb{R}} \operatorname{Hom}\left(\pi_{1}(X), \mathbb{R}\right)$ is an upper bound for the deficiency up to DTC of $\pi_{1}(\tilde{M}, u)$. Here the deficiency up to DTC is the maximum of $m-r$, where $m$ is the cardinal of a generating family, and $r$ of the associated relations, both up to DTC.

5.3. Comparing Novikov fundamental group and homologies. In this section, we use the results of the two previous sections to provide examples where the numerical estimates for the number of index 1 and 2 critical points obtained from the Novikov fundamental group are essentially different from the estimates that can be derived from the Novikov homologies.

5.3.1. Comparison on the minimal integration cover. Suppose now that $X$ satisfies the following conditions

$$
\begin{gathered}
\operatorname{dim} X=n \geq 4, \\
H_{1}(X, \mathbb{Z})=H_{2}(X, \mathbb{Z})=0, \\
\pi_{1}(X) \neq 1 .
\end{gathered}
$$

This is the case for instance if $X=S \times \mathbb{S}^{n-3}, n \geq 4$, where $S$ is the Poincaré homology sphere.

Recall that the manifold under interest is then $M=\mathbb{T}^{n} \times X$, endowed with the one form $u=\left[\pi^{*}\left(d \theta_{1}\right)\right]$, and $\tilde{M}$ is the minimal integration cover of $u$.

Then from proposition 5.1, we get

$$
H N_{1}(\tilde{M}, u)=H N_{2}(\tilde{M}, u)=0,
$$

while from proposition 5.4 we get

$$
\pi_{1}(\tilde{M}, u) \neq 1 \text {. }
$$

In particular, the Novikov homology on the minimal cover does not detect any index 1 or 2 critical points, while the Novikov fundamental group does since we necessarily have

$$
\mu_{D T C}(\Pi)>0 .
$$

Similarly, if $G$ has non trivial relations, which is the case for the Poincaré sphere for instance, then so does $\pi_{1}(\tilde{M}, u)$ and hence

$$
\rho_{D T C}(\Pi)>0 \text {. }
$$


In the case where $X=S \times \mathbb{S}^{n-3}$, we can be even more precise, since $\pi_{1}(X)$ is not cyclic and has at least 2 generators, we have

$$
\mu_{D T C}\left(\pi_{1}(\tilde{M}, u)\right)=2 .
$$

Similarly (5.1) is a presentation of $\pi_{1}(X)$ with 2 generators and 2 relations, so $\rho_{D T C}\left(\pi_{1}(\tilde{M}, u)\right) \leq 2$, while it is not hard to see from these relations that $\operatorname{dim}_{\mathbb{R}} \operatorname{Hom}\left(\pi_{1}(X), \mathbb{R}\right)=0$, so that $\rho_{D T C}\left(\pi_{1}(\tilde{M}, u)\right) \geq 2$ and finally

$$
\rho_{D T C}\left(\pi_{1}(\tilde{M}, u)\right)=2 .
$$

This proves Theorem 1.5 which we recall below

Corollary 5.15. When $X=S \times \mathbb{S}^{n-3}$, the Novikov fundamental group associated to the class $u=\pi^{*}[d \theta]$ on $M=\mathbb{T}^{n} \sharp X$ is non trivial, and any Morse 1-form in this cohomology class has to have at least 2 index 1 and 2 index 2 critical points.

This example shows that the lower bounds (3.2) and (3.3) derived from Novikov fundamental group are essentially different from the Novikov inequalities derived from the Novikov homology associated to the minimal integration cover.

Remark 19. When $X=S \times \mathbb{S}^{n-3}$, we also have $\pi_{2}(X)=0$, and hence $H N_{2}\left(M, u ; \mathbb{Z}\left[\pi_{1}(M)\right]\right)=0$, so that the Morse-Novikov inequalities for the universal cover do not detect any index 2 critical points either.

However, the comparison of the Novikov homologies on the universal and minimal integration covers still allow to detect index 2 critical points as detailed below.

5.3.2. Comparison on the universal cover. A nice observation pointed to us by A. Pajitnov allows to detect index 2 critical points from the comparison of the Novikov homology on the minimal and universal covers, even though the second homology group vanish : starting with a 1-form $\alpha$ in the class $u$, it is possible to modify to remove all the index 0 critical points, without creating new index 2 critical points. Then, since $H N_{1}\left(M, u ; \mathbb{Z}\left[\pi_{1}\right]\right) \neq 0, \alpha$ has to have index 1 critical points. Moreover, since $H N_{1}(M, u)=0$, all these points have to be killed by index 2 critical points.

The number of index 2 critical points detected by this observation is at most that of index 1 critical points. More precisely, it is at most the difference $\left|\tilde{\beta}_{1}-\beta_{1}\right|$ where $\beta_{1}$ and $\tilde{\beta}_{1}$ are the minimal number of generators for $H N_{1}(M, u)$ and $H N_{1}\left(M, u ; \mathbb{Z}\left[\pi_{1}(M)\right]\right)$.

Consider the case where $M=\mathbb{T}^{n} \sharp X$ with $X=\mathbb{R P}^{n}(n \geq 4)$.

From (5.1), we obtain

$$
H N_{1}(M, u)=\mathbb{Z} / 2 \mathbb{Z}((t)) \quad \text { and } \quad H N_{2}(M, u)=0 .
$$

On the other hand, $H N_{1}\left(M, u ; \mathbb{Z}\left[\pi_{1}(M)\right]\right) \neq 0$ so that from $[5.2$, we get that the minimal number of generators of $H N_{1}\left(M, u ; \mathbb{Z}\left[\pi_{1}(M)\right]\right)$ is 1 , and

$$
H N_{2}\left(M, u ; \mathbb{Z}\left[\pi_{1}(M)\right]\right)=0 .
$$


In particular, the comparison of the Novikov homologies associated to the minimal and universal covers does not provide any index 2 critical points, while the Novikov fundamental group does, since we obviously have

$$
\mu_{D T C}\left(\pi_{1}(M, u)\right)=1 \text { and } \rho_{D T C}\left(\pi_{1}(M, u)\right)=1 .
$$

In particular, every Morse 1-form on $\mathbb{T}^{n} \sharp \mathbb{R} \mathbb{P}^{n}$ has to have at least one index 2 critical point.

Acknowledgement. HVL thanks JFB and Nguyên Tiên Zung, Lê Ngoc Mai for their invitation to Toulouse and their hospitality during her visit in May 2016, when she and JFB started this project aiming to develop ideas in Barraud2014 further for the Novikov case.

AG thanks JFB and CIMI for the invitation to Toulouse in June 2017, when she joined the project, the Hausdorff Research Institute for Mathematics (HIM), University of Bonn for its support and hospitality in the fall 2017, and the Mathematics Institute of Uppsala University for its nice working atmosphere.

We thank Andrei Pajitnov for his stimulating comments to the first eprint of our paper.

\section{REFERENCES}

[AD2014] M. Audin And M. Damian, Morse theory and Floer Homology, Springer Verlag 2014.

[Barraud2014] J.-F. Barraud, A Floer fundamental group, to appear in Annales de l'École normale supérieure.

[Damian2009] M. DAmiAn, Constraints on exact Lagrangians in cotangent bundles of manifolds fibered over the circle. Comment. Math. Helv. 84(4), 705-746 (2009).

[DnF1984] B. A. Dubrovin, S. P. Novikov And A. T. Fomenko, Modern Geometry - Methods and Applications, part III, Introduction to Homology theory, Moscow, Nauka 1984, translation in English, Springer, 1990.

[Gadbled2009] A. GADBled, Obstructions to the existence of monotone Lagrangian embeddings into cotangent bundles of manifolds fibered over the circle, Annales de l'Institut Fourier, 59 (2009), no. 3, pp 1135-1175.

[Farber2004] M. FARBER, Topology of closed 1-forms, AMS, 2004.

[FGS2010] M. Farber, R. GeÏGan, D. Shyutts, Closed 1-forms in topology and geometric group theory, Uspekhi Mat. Nauk 65 (2010), no. 1(391), pp 145-176; translation in Russian Math. Surveys 65 (2010), no. 1, pp 143-172

[FarberSchutz2007] M. FARBER AND D.SCHÜTz, Moving homology classes to infinity. Forum Math. 19 (2007), no. 2, 281-296.

[Latour1994] F. LATOUR, Existence de 1-formes fermées non singulières dans une classe de cohomologie de de Rham, Pub. Math. de l'I.H.E.S., 80 (1994), 135-194.

[LO1995] H. V. LÊ AND K. ONO, Symplectic fixed points, the Calabi invariant and Novikov homology, Topology 34 (1995), 155-176.

[Livingston] Livingston, Charles, Four-manifolds of large negative deficiency, Math. Proc. Cambridge Philos. Soc. 138 (2005), no. 1, 107-115.

[Massey1978] W. S. MAssey, Homology and cohomology theories, Marcel Dekker, New York 1978.

[Novikov1981] S. P. Novikov, Many-valued functions and functionals. An analogue of Morse theory, Sov. Math. Dokl. 24 (1981), pp. 222-226.

[Pajitnov2006] A. PAJitnov, Circle-valued Morse Theory, Walter de Gruyter, 2006. 
[Sharko1993] V. V. Sharko, Functions on Manifolds, AMS, Translations of Mathematical Monographs, 1993.

[Sikorav1986] J.-C. SikORAV, Un problème de disjonction par isotopie symplectique dans un fibré cotangent, Annales scientifiques de l'École Normale Supérieure, Série 4 : Tome 19 (1986) no. 4 , 543-552.

[Sikorav1987] J.-C. Sikorav, Points fixes de difféomorphismes symplectiques, intersections de sous-variétés lagrangiennes, et singularités de un-formes fermées, Thése de Doctorat d'Etat Es Sciences Mathématiques, Université Paris-Sud, Centre d'Orsay, 1987.

[Sikorav2016] J.-C. Sikorav, On Novikov homology, preprint 2016.

[Tischler1970] D. Tischler, On fibering certain foliated manifolds over $S^{1}$, Topology, vol 9 (1970), 153-154.

[Usher2008] Usher, Michael, Spectral numbers in Floer theories. Compos. Math. 144 (2008), no. 6, 1581-1592.

E-mail address: barraud@math.univ-toulouse.fr

Institut de mathématiques de Toulouse, Université Paul Sabatier - Toulouse III, 118 route de Narbonne, F-31062 Toulouse Cedex 9, France

E-mail address: agnes.gadbled@math.uu.se

Department of Mathematics, Uppsala University, Box 480, 75106 Uppsala, SWEDEN

E-mail address: rgolovko@ulb.ac.be

Départment de Mathématique, Université libre de Bruxelles, CP 218, Boulevard Du Triomphe, B-1050 Bruxelles, Belgique

E-mail address: hvle@math.cas.cz

Institute of Mathematics CAS, Zitna 25, 11567 Praha 1, Czech Republic 